\title{
Variational method for multiple parameter identification in elliptic PDEs
}

\author{
Tran Nhan Tam Quyen[
}

Department of Mathematics, University of Hamburg, Bundesstraße 55, D-20146 Hamburg, Germany Institut für Numerische und Angewandte Mathematik, Universität Göttingen, Lotzestraße 16-18, D-37083 Göttingen, Germany

\begin{abstract}
In the present paper we investigate the inverse problem of identifying simultaneously the diffusion matrix, source term and boundary condition in the Neumann boundary value problem for an elliptic partial differential equation (PDE) from a measurement data, which is weaker than required of the exact state. A variational method based on energy functions with Tikhonov regularization is here proposed to treat the identification problem. We discretize the PDE with the finite element method and prove the convergence as well as analyse error bounds of this approach.
\end{abstract}

Key words and phrases: Multiple parameter identification, diffusion matrix, source term, boundary condition, ill-posed problem, finite element method.

AMS Subject Classifications: 65N21, 65N12, 35J25, 35R30

\section{Introduction}

Let $\Omega$ be an open bounded connected domain of $R^{d}, 1 \leq d \leq 3$ with polygonal boundary $\partial \Omega$. In this paper we study the problem of identifying simultaneously the diffusion matrix $Q$, source term $f$ and boundary condition $g$ as well as the state $\Phi$ in the Neumann boundary value problem for the elliptic PDE

$$
\begin{aligned}
-\nabla \cdot(Q \nabla \Phi) & =f \quad \text { in } \quad \Omega, \\
Q \nabla \Phi \cdot \vec{n} & =g \text { on } \partial \Omega
\end{aligned}
$$

from a measurement $z_{\delta} \in L^{2}(\Omega)$ of the solution $\Phi \in H^{1}(\Omega)$, where $\vec{n}$ is the unit outward normal on $\partial \Omega$.

To formulate precisely our problem, let us first denote by $\mathcal{S}_{d}$ the set of all symmetric, real $d \times d$-matrices equipped with the inner product $M \cdot N:=\operatorname{trace}(M N)$ and the corresponding norm $\|M\|_{\mathcal{S}_{d}}=(M \cdot M)^{1 / 2}=$ $\left(\sum_{i, j=1}^{d} m_{i j}^{2}\right)^{1 / 2}$, where $M:=\left(m_{i j}\right)_{i, j=\overline{1, d}}$. Furthermore, for $1 \leq p \leq \infty$ we set

$$
\mathbf{L}_{\mathrm{sym}}^{p}(\Omega):=\left\{H:=\left(h_{i j}\right)_{i, j=\overline{1, d}} \in L^{p}(\Omega)^{d \times d} \mid H(x):=\left(h_{i j}(x)\right)_{i, j=\overline{1, d}} \in \mathcal{S}_{d} \quad \text { a.e. in } \quad \Omega\right\} .
$$

In $\mathbf{L}_{\text {sym }}^{2}(\Omega)$ we use the scalar product $\left(H^{1}, H^{2}\right)_{\mathbf{L}_{\text {sym }}^{2}(\Omega)}=\sum_{i, j=1}^{d}\left(h_{i j}^{1}, h_{i j}^{2}\right)_{L^{2}(\Omega)}$ and the corresponding norm $\|H\|_{\mathbf{L}_{\text {sym }}^{2}(\Omega)}:=\left(\sum_{i, j=1}^{d}\left\|h_{i j}\right\|_{L^{2}(\Omega)}^{2}\right)^{1 / 2}=\left(\int_{\Omega}\|H(x)\|_{\mathcal{S}_{d}}^{2} d x\right)^{1 / 2}$, while the space $\mathbf{L}_{\text {sym }}^{\infty}(\Omega)$ is endowed with the norm $\|H\|_{\mathbf{L}_{\mathrm{sym}}^{\infty}(\Omega)}:=\max _{i, j=\overline{1, d}}\left\|h_{i j}\right\|_{L^{\infty}(\Omega)}$.

Let us denote by

$$
\mathcal{H}_{a d}:=\mathcal{Q}_{a d} \times \mathcal{F}_{a d} \times \mathcal{G}_{a d}
$$

with

$$
\begin{aligned}
\mathcal{Q}_{a d} & :=\left\{\left.Q \in \mathbf{L}_{\text {sym }}^{\infty}(\Omega) \quad|\underline{q}| \xi\right|^{2} \leq Q(x) \xi \cdot \xi \leq \bar{q}|\xi|^{2} \quad \text { for all } \xi \in R^{d}\right\} \\
\mathcal{F}_{a d} & :=L^{2}(\Omega) \\
\mathcal{G}_{a d} & :=L^{2}(\partial \Omega)
\end{aligned}
$$

Email: quyen.tran@uni-hamburg.de, quyen.tran@uni-goettingen.de

The author gratefully acknowledges support of the Alexander von Humboldt-Foundation and the Lothar Collatz Center for Computing in Science at the University of Hamburg, and the University of Goettingen, State of Lower Saxony, Germany 
and $\underline{q}, \bar{q}$ being given constants satisfying $\bar{q} \geq \underline{q}>0$. Let

$$
\gamma: H^{1}(\Omega) \quad \rightarrow \quad H^{1 / 2}(\partial \Omega)
$$

be the continuous Dirichlet trace operator and $H_{\diamond}^{1}(\Omega)$ be the closed subspace of $H^{1}(\Omega)$ consisting all functions with zero-mean on the boundary, i.e.

$$
H_{\diamond}^{1}(\Omega):=\left\{u \in H^{1}(\Omega) \quad \mid \int_{\partial \Omega} \gamma u d x=0\right\}
$$

while $C_{\Omega}$ stands for the positive constant appearing in the Poincaré-Friedrichs inequality (cf. [38])

$$
C_{\Omega} \int_{\Omega} \varphi^{2} d x \leq \int_{\Omega}|\nabla \varphi|^{2} d x \text { for all } \varphi \in H_{\diamond}^{1}(\Omega) .
$$

Then, due to the coervicity condition

$$
\|\varphi\|_{H^{1}(\Omega)}^{2} \leq \frac{1+C_{\Omega}}{C_{\Omega}} \int_{\Omega}|\nabla \varphi|^{2} d x \leq \frac{1+C_{\Omega}}{C_{\Omega} \underline{q}} \int_{\Omega} Q \nabla \varphi \cdot \nabla \varphi d x
$$

holding for all $\varphi \in H_{\diamond}^{1}(\Omega), Q \in \mathcal{Q}_{a d}$ and the Lax-Milgram lemma, we conclude for each $(Q, f, g) \in \mathcal{H}_{a d}$, there exists a unique weak solution $\Phi$ of $1.1-1.2$ in the sense that $\Phi \in H_{\diamond}^{1}(\Omega)$ and satisfies the identity

$$
\int_{\Omega} Q \nabla \Phi \cdot \nabla \varphi d x=(f, \varphi)+\langle g, \gamma \varphi\rangle
$$

for all $\varphi \in H_{\diamond}^{1}(\Omega)$. Here the expressions $(\cdot, \cdot)$ and $\langle\cdot, \cdot\rangle$ stand for the scalar product on space $L^{2}(\Omega)$ and $L^{2}(\partial \Omega)$, respectively. Furthermore, there holds the priori estimate

$$
\begin{aligned}
\|\Phi\|_{H^{1}(\Omega)} & \leq \frac{1+C_{\Omega}}{C_{\Omega} \underline{q}}\left(\|\gamma\|_{\mathcal{L}\left(H^{1}(\Omega), H^{1 / 2}(\partial \Omega)\right.}\|g\|_{L^{2}(\partial \Omega)}+\|f\|_{L^{2}(\Omega)}\right) \\
& \leq C_{\mathcal{N}}\left(\|g\|_{L^{2}(\partial \Omega)}+\|f\|_{L^{2}(\Omega)}\right)
\end{aligned}
$$

with

$$
C_{\mathcal{N}}:=\frac{1+C_{\Omega}}{C_{\Omega} \underline{q}} \max \left(1,\|\gamma\|_{\mathcal{L}\left(H^{1}(\Omega), H^{1 / 2}(\partial \Omega)\right)}\right) .
$$

Then we can define the non-linear coefficient-to-solution operator

$$
\mathcal{U}: \quad \mathcal{H}_{a d} \rightarrow H_{\diamond}^{1}(\Omega)
$$

which maps each $(Q, f, g) \in \mathcal{H}_{a d}$ to the unique weak solution $\mathcal{U}_{Q, f, g}:=\Phi$ of the problem (1.1)-(1.2). Here, for convenience in computing numerical solutions of the pure Neumann problem we normalize the solution with vanishing mean on the boundary (cf., e.g., [23, Subsection 5.2], 28, Section 2]); however, all results performed in the present paper are still valid for the normalization of solutions of the Neumann problem with zero-mean over the domain, i.e. $\mathcal{U}_{Q, f, g} \in\left\{u \in H^{1}(\Omega) \mid \int_{\Omega} u d x=0\right\}$. The identification problem is now stated as follows:

$$
\begin{gathered}
\text { Given } \Phi^{\dagger}:=\mathcal{U}_{Q, f, q} \in H_{\diamond}^{1}(\Omega), \text { find an element }(Q, f, g) \in \mathcal{H}_{a d} \\
\text { such that (1.6) is satisfied with } \Phi^{\dagger} \text { and } Q, f, g .
\end{gathered}
$$

This inverse problem may have more than one solution and it is highly ill-posed. In fact, assume that the exact $\Phi^{\dagger} \in C_{c}^{2}(\Omega)$, the space of all functions having second-order derivatives with compact support in $\Omega$. Then, for all $Q \in C^{1}(\Omega)^{d \times d} \cap \mathcal{Q}_{a d}$ the element $(\bar{Q}, \bar{f}, \bar{g}):=\left(Q,-\nabla\left(Q \cdot \nabla \Phi^{\dagger}\right), 0\right)$ is a solution of the above identification problem, i.e. $\mathcal{U}_{\bar{Q}, \bar{f}, \bar{g}}=\Phi^{\dagger}$. In other words we are considering to solve an equation $\mathcal{U}_{Q, f, g}=\Phi^{\dagger}$, where the forward operator $\mathcal{U}$ is non-linear and non-injective. Without using additional objective a-priori information or without exploiting other observation data as considering here, it is difficult for us to classify sought targets. Following the general convergence theory for ill-posed problems (see, e.g., [9, Chapter 5] and 
[43, Subsection 3.2.1], or the classical monograph [15, Section 10.1]), in the present paper we are interested in finding exact solutions with penalty minimizing, which is defined as

$$
\left(Q^{\dagger}, f^{\dagger}, g^{\dagger}\right):=\arg \min _{(Q, f, g) \in \mathcal{I}\left(\Phi^{\dagger}\right)} \mathcal{R}(Q, f, g)
$$

where $\mathcal{I}\left(\Phi^{\dagger}\right):=\left\{(Q, f, g) \in \mathcal{H}_{a d} \mid \mathcal{U}_{Q, f, g}=\Phi^{\dagger}\right\}$ and the penalty term

$$
\mathcal{R}(Q, f, g):=\|Q\|_{\mathbf{L}_{\text {sym }}^{2}(\Omega)}^{2}+\|f\|_{L^{2}(\Omega)}^{2}+\|g\|_{L^{2}(\partial \Omega)}^{2} .
$$

We note that the admissible set $\mathcal{I}\left(\Phi^{\dagger}\right)$ of the problem 1.8$)$ is non-empty, convex and weakly closed in $\mathbf{L}_{\text {sym }}^{2}(\Omega) \times L^{2}(\Omega) \times L^{2}(\partial \Omega)$, so that the minimizer $\left(Q^{\dagger}, f^{\dagger}, g^{\dagger}\right)$ is defined uniquely. Furthermore, the exact data $\Phi^{\dagger}$ may not be known in practice, thus we assume instead of $\Phi^{\dagger}$ to have a measurement $z_{\delta} \in L^{2}(\Omega)$ such that

$$
\left\|\Phi^{\dagger}-z_{\delta}\right\|_{L^{2}(\Omega)} \leq \delta \text { with } \delta>0 .
$$

Our identification problem is now to reconstruct $\left(Q^{\dagger}, f^{\dagger}, g^{\dagger}\right) \in \mathcal{H}_{a d}$ from $z_{\delta}$.

Let $\left(\mathcal{T}^{h}\right)_{0<h<1}$ denote a family of triangulations of the domain $\bar{\Omega}$ with the mesh size $h$ and $\mathcal{U}^{h}$ be the approximation of the operator $\mathcal{U}$ on the piecewise linear, continuous finite element space associated with $\mathcal{T}^{h}$. Furthermore, let $\Pi^{h}$ be the Clément's mollification interpolation operator (cf. \$2). The standard method for solving the above mentioned identification problem is the output least squares one with Tikhonov regularization, i.e. one considers a minimizer of the problem

$$
\min _{(Q, f, g) \in \mathcal{H}_{a d}}\left\|\mathcal{U}_{Q, f, g}^{h}-\Pi^{h} z_{\delta}\right\|_{L^{2}(\Omega)}^{2}+\rho \mathcal{R}(Q, f, g)
$$

as a discrete approximation of the identified coefficient $\left(Q^{\dagger}, f^{\dagger}, g^{\dagger}\right)$, here $\rho>0$ is the regularization parameter. However, due to the non-linearity of the coefficient-to-solution operator, we are faced with certain difficulties in holding the non-convex minimization problem (1.10). Thus, instead of working with the above least squares functional and following the use of energy functions (cf. [37, 35, 48]), in the present work the convex cost function (cf. $\$ 2$ )

$$
(Q, f, g) \in \mathcal{H}_{a d} \quad \mapsto \mathcal{J}_{\delta}^{h}(Q, f, g):=\int_{\Omega} Q \nabla\left(\mathcal{U}_{Q, f, g}^{h}-\Pi^{h} z_{\delta}\right) \cdot \nabla\left(\mathcal{U}_{Q, f, g}^{h}-\Pi^{h} z_{\delta}\right) d x
$$

will be taken into account. We then consider a unique minimizer $\left(Q^{h}, f^{h}, g^{h}\right)$ of the strictly convex problem

$$
\min _{(Q, f, g) \in \mathcal{H}_{a d}} \mathcal{J}_{\delta}^{h}(Q, f, g)+\rho \mathcal{R}(Q, f, g)
$$

as a discrete regularized solution of the identification problem. Note that, by using variational discretization concept introduced in [22, every solution of the minimization problem (1.11) is proved to automatically belong to finite dimensional spaces. Thus, a discretization of the admissible set $\mathcal{H}_{a d}$ can be avoided. Furthermore, for simplicity of exposition we here restrict ourselves to the case of one set of data $\left(z_{\delta}\right)_{\delta>0}$. In case with several sets of data $\left(z_{\delta_{i}}\right)_{i=1}^{I}$ being available, we can replace the misfit term in the problem (1.11) by the term $\frac{1}{I} \sum_{i=1}^{I} \mathcal{J}_{\delta_{i}}^{h}(Q, f, g)$.

In $\S 3$ we will show the convergence of these approximation solutions $\left(Q^{h}, f^{h}, g^{h}\right)$ to the identification $\left(Q^{\dagger}, f^{\dagger}, g^{\dagger}\right)$ in the $\mathbf{L}_{\text {sym }}^{2}(\Omega) \times L^{2}(\Omega) \times L^{2}(\partial \Omega)$-norm as well as the convergence of corresponding approximation states $\left(\mathcal{U}_{Q^{h}, f^{h}, g^{h}}\right)$ to the exact $\Phi^{\dagger}$ in the $H^{1}(\Omega)$-norm. Under the structural source condition - but without the smallness requirement - of the general convergence theory for non-linear, ill-posed problems (cf. [15, 16]), we prove in $\$$ error bounds for these discrete approximations. For the numerical solution of the minimization problem (1.11) we in $\$ 5$ employ a gradient projection algorithm with Armijo steplength rule. Finally, a numerical implementation will be performed to illustrate the theoretical findings.

The coefficient identification problem in PDEs arises from different contexts of applied sciences, e.g., from aquifer analysis, geophysical prospecting and pollutant detection, and attracted great attention from many scientists in the last 30 years or so. For surveys on the subject one may consult in [3, 9, 29, 43, 45, 46]. 
The problem of identifying the scalar diffusion coefficient has been extensively studied for both theoretical research and numerical implementation, see e.g., [7, 8, 10, 11, 17, 18, 19, 27, 30, 32, 33, 36, 40, 48, Some contributions for the case of the simultaneous identification can be found in [2, 20, 21, 34] while some works treated the diffusion matrix case have been obtained in [14, 24, 25, 26, 39].

We conclude this introduction with the following mention. By using the H-convergent concept, the convergence analysis presented in 24] can not be applied directly to the problem of identifying scalar diffusion coefficients. There are two main difficulties for the scalar coefficient identification. First, the set

$$
\mathcal{D}:=\left\{q I_{d} \mid q \in L^{\infty}(\Omega) \text { with } \underline{q} \leq q(x) \leq \bar{q} \quad \text { a.e. in } \quad \Omega \quad \text { and } \quad I_{d} \text { is the unit } d \times d \text {-matrix }\right\}
$$

is in general not a closed subset of $\mathcal{Q}_{a d}$ under the topology of the H-convergence (cf. [47]), i.e. if the sequence $\left(q_{n} I_{d}\right)_{n} \subset \mathcal{D}$ is H-convergent to $Q \in \mathcal{Q}_{a d}$, then $Q$ is not necessarily proportional to $I_{d}$ in dimension $d \geq 2$ or $Q \notin \mathcal{D}$. Second, the forward operator $\mathcal{U}$ is not weakly sequentially closed in $L^{2}$, i.e. if $\left(q_{n}, \mathcal{U}\left(q_{n}\right)\right) \rightarrow(q, \mathcal{Y})$ weakly in $L^{2}(\Omega) \times L^{2}(\Omega)$, it is not guaranteed that $\mathcal{Y}=\mathcal{U}(q)$ (see [14] and the references therein for counterexamples). To overcome these difficulties, a different analysis technique based on the convexity of the cost functional will be taken into counting. Due to the weak* closedness of the set $\mathcal{D}$ above in $\mathbf{L}_{\text {sym }}^{\infty}(\Omega)$ (cf. Remark 2.1), the convergence analysis performed in the present paper thus covers the scalar diffusion identification case. On the other hand, in 24] the source term and the boundary condition were assumed to be given. In the present situation they are variables which have to be found simultaneously together with the diffusion from observations.

Throughout the paper we write $\int_{\Omega} \cdots$ instead of $\int_{\Omega} \cdots d x$ for the convenience of relevant notations. We use the standard notion of Sobolev spaces $H^{1}(\Omega), H^{2}(\Omega), W^{k, p}(\Omega)$, etc from, e.g., [1].

\section{Finite element discretization}

\subsection{Preliminaries}

In product spaces $\mathbf{L}_{\text {sym }}^{2}(\Omega) \times L^{2}(\Omega) \times L^{2}(\partial \Omega)$ and $\mathbf{L}_{\text {sym }}^{\infty}(\Omega) \times L^{2}(\Omega) \times L^{2}(\partial \Omega)$ we use respectively the norm

$$
\begin{aligned}
\|(H, l, s)\|_{\mathbf{L}_{\mathrm{sym}}^{2}(\Omega) \times L^{2}(\Omega) \times L^{2}(\partial \Omega)} & =\left(\|H\|_{\mathbf{L}_{\mathrm{sym}}^{2}(\Omega)}^{2}+\|l\|_{L^{2}(\Omega)}^{2}+\|s\|_{L^{2}(\partial \Omega)}^{2}\right)^{1 / 2} \text { and } \\
\|(H, l, s)\|_{\mathbf{L}_{\mathrm{sym}}^{\infty}(\Omega) \times L^{2}(\Omega) \times L^{2}(\partial \Omega)} & =\|H\|_{\mathbf{L}_{\mathrm{sym}}^{\infty}(\Omega)}+\|l\|_{L^{2}(\Omega)}+\|s\|_{L^{2}(\partial \Omega)} .
\end{aligned}
$$

We note that the coefficient-to-solution operator

$$
\mathcal{U}: \quad \mathcal{H}_{a d} \subset \mathbf{L}_{\text {sym }}^{\infty}(\Omega) \times L^{2}(\Omega) \times L^{2}(\partial \Omega) \rightarrow H_{\diamond}^{1}(\Omega)
$$

with

$$
\Gamma:=(Q, f, g) \in \mathcal{H}_{a d} \rightarrow \mathcal{U}(\Gamma):=\mathcal{U}_{\Gamma}
$$

is Fréchet differentiable on $\mathcal{H}_{a d}$. For each $\Gamma=(Q, f, g) \in \mathcal{H}_{a d}$ the action of its Fréchet derivative in direction $\lambda:=(H, l, s) \in \mathbf{L}_{\text {sym }}^{\infty}(\Omega) \times L^{2}(\Omega) \times L^{2}(\partial \Omega)$ denoted by $\xi_{\lambda}:=\mathcal{U}_{\Gamma}^{\prime}(\lambda):=\mathcal{U}^{\prime}(\Gamma)(\lambda)$ is the unique weak solution in $H_{\diamond}^{1}(\Omega)$ to the equation

$$
\int_{\Omega} Q \nabla \xi_{\lambda} \cdot \nabla \varphi=-\int_{\Omega} H \nabla \mathcal{U}_{\Gamma} \cdot \nabla \varphi+(l, \varphi)+\langle s, \gamma \varphi\rangle
$$

for all $\varphi \in H_{\diamond}^{1}(\Omega)$.

In $\mathcal{S}_{d}$ we introduce the convex subset

$$
\mathcal{K}:=\left\{M \in \mathcal{S}_{d} \quad \mid \underline{q} \leq M \xi \cdot \xi \leq \bar{q} \text { for all } \xi \in R^{d}\right\}
$$

together with the orthogonal projection $P_{\mathcal{K}}: \mathcal{S}_{d} \rightarrow \mathcal{K}$ that is characterised by

$$
\left(A-P_{\mathcal{K}}(A)\right) \cdot\left(B-P_{\mathcal{K}}(A)\right) \leq 0
$$


for all $A \in \mathcal{S}_{d}$ and $B \in \mathcal{K}$. Furthermore, let $\xi:=\left(\xi_{1}, \cdots, \xi_{d}\right)$ and $\eta:=\left(\eta_{1}, \cdots, \eta_{d}\right)$ be two arbitrary vectors in $R^{d}$, we use the notation

$$
(\xi \otimes \eta)_{1 \leq i, j \leq d} \in \mathcal{S}_{d} \quad \text { with } \quad(\xi \otimes \eta)_{i j}:=\frac{1}{2}\left(\xi_{i} \eta_{j}+\xi_{j} \eta_{i}\right) \quad \text { for all } \quad i, j=1, \cdots, d .
$$

We close this subsection by the following note.

Remark 2.1. Let

$$
\mathbf{D}:=\left\{q \in L^{\infty}(\Omega) \quad \mid \quad \underline{q} \leq q(x) \leq \bar{q} \quad \text { a.e. in } \quad \Omega\right\} .
$$

Then $\mathbf{D}$ is a weakly* compact subset of $L^{\infty}(\Omega)$, i.e. for any sequence $\left(q_{n}\right)_{n} \subset \mathbf{D}$ a subsequence $\left(q_{n_{m}}\right)_{m}$ and an element $\xi_{\infty} \in \mathbf{D}$ exist such that $\left(q_{n_{m}}\right)_{m}$ is weakly* convergent in $L^{\infty}(\Omega)$ to $\xi_{\infty}$. In other words, for all $\theta_{1} \in L^{1}(\Omega)$ there holds the limit

$$
\lim _{m \rightarrow \infty} \int_{\Omega} q_{n_{m}} \theta_{1}=\int_{\Omega} \xi_{\infty} \theta_{1}
$$

We also remark that any $\Psi \in L^{\infty}(\Omega)$ can be considered as an element in $L^{\infty}(\Omega)^{*}$ by

$$
\langle\Psi, \psi\rangle_{\left(L^{\infty}(\Omega)^{*}, L^{\infty}(\Omega)\right)}:=\int_{\Omega} \Psi \psi
$$

for all $\psi$ in $L^{\infty}(\Omega)$ and $\|\Psi\|_{L^{\infty}(\Omega)^{*}} \leq|\Omega| \cdot\|\Psi\|_{L^{\infty}(\Omega)}$. Therefore, due to 2.2 , the assertion of Remark 2.1 is a direct consequence of the Banach-Alaoglu theorem.

\subsection{Discretization}

Let $\left(\mathcal{T}^{h}\right)_{0<h<1}$ be a family of regular and quasi-uniform triangulations of the domain $\bar{\Omega}$ with the mesh size $h$ such that each vertex of the polygonal boundary $\partial \Omega$ is a node of $\mathcal{T}_{h}$. For the definition of the discretization space of the state functions let us denote

$$
\mathcal{V}_{1}^{h}=\left\{\varphi^{h} \in C(\bar{\Omega}) \cap H_{\diamond}^{1}(\Omega) \quad \mid \quad \varphi^{h}{ }_{\mid T} \in \mathcal{P}_{1}(T) \quad \text { for all } T \in \mathcal{T}^{h}\right\}
$$

with $\mathcal{P}_{r}$ consisting all polynomial functions of degree at most $r$. Similar to the continuous case, we have the following result.

Lemma 2.2. Let $(Q, f, g)$ be in $\mathcal{H}_{a d}$. Then the variational equation

$$
\int_{\Omega} Q \nabla \Phi^{h} \cdot \nabla \varphi^{h}=\left(f, \varphi^{h}\right)+\left\langle g, \gamma \varphi^{h}\right\rangle
$$

for all $\varphi^{h} \in \mathcal{V}_{1}^{h}$ admits a unique solution $\Phi^{h} \in \mathcal{V}_{1}^{h}$. Furthermore, the priori estimate

$$
\left\|\Phi^{h}\right\|_{H^{1}(\Omega)} \leq C_{\mathcal{N}}\left(\|f\|_{L^{2}(\Omega)}+\|g\|_{L^{2}(\partial \Omega)}\right)
$$

is satisfied.

The map $\mathcal{U}^{h}: \mathcal{H}_{a d} \subset \mathbf{L}_{\text {sym }}^{\infty}(\Omega) \times L^{2}(\Omega) \times L^{2}(\partial \Omega) \rightarrow \mathcal{V}_{1}^{h}$ from each $\Gamma:=(Q, f, g) \in \mathcal{H}_{\text {ad }}$ to the unique solution $\mathcal{U}_{\Gamma}^{h}:=\Phi^{h}$ of (2.4) is called the discrete coefficient-to-solution operator. This operator is also Fréchet differentiable on the set $\mathcal{H}_{a d}$. For each $\Gamma=(Q, f, g) \in \mathcal{H}_{a d}$ and $\lambda:=(H, l, s) \in \mathbf{L}_{\text {sym }}^{\infty}(\Omega) \times L^{2}(\Omega) \times L^{2}(\partial \Omega)$ the Fréchet differential $\xi_{\lambda}^{h}:=\mathcal{U}_{\Gamma}^{h^{\prime}}(\lambda)$ is an element of $\mathcal{V}_{1}^{h}$ and satisfies for all $\varphi^{h}$ in $\mathcal{V}_{1}^{h}$ the equation

$$
\int_{\Omega} Q \nabla \xi_{\lambda}^{h} \cdot \nabla \varphi^{h}=-\int_{\Omega} H \nabla \mathcal{U}_{\Gamma}^{h} \cdot \nabla \varphi^{h}+\left(l, \varphi^{h}\right)+\left\langle s, \gamma \varphi^{h}\right\rangle .
$$

Due to the standard theory of the finite element method for elliptic problems (cf. [6, 12]), for any fixed $\Gamma=(Q, f, g) \in \mathcal{H}_{a d}$ there holds the limit

$$
\lim _{h \rightarrow 0}\left\|\mathcal{U}_{\Gamma}-\mathcal{U}_{\Gamma}^{h}\right\|_{H^{1}(\Omega)}=0 .
$$


Let

$$
\Pi^{h}: \quad L^{1}(\Omega) \rightarrow\left\{\varphi^{h} \in C(\bar{\Omega}) \quad \mid \quad \varphi^{h}{ }_{\mid T} \in \mathcal{P}_{1}(T) \quad \text { for all } \quad T \in \mathcal{T}^{h}\right\}
$$

be the Clément's mollification interpolation operator with properties

$$
\lim _{h \rightarrow 0}\left\|\phi-\Pi^{h} \phi\right\|_{H^{k}(\Omega)}=0 \quad \text { for all } \quad k \in\{0,1\}
$$

and

$$
\left\|\phi-\Pi^{h} \phi\right\|_{H^{k}(\Omega)} \leq C h^{l-k}\|\phi\|_{H^{l}(\Omega)}
$$

for $0 \leq k \leq l \leq 2$, where $C$ is independent of $h$ and $\phi$ (cf. [13, 4, 5, 44). Then, using the discrete operator $\mathcal{U}^{h}$ and the interpolation operator $\Pi^{h}$, we can now introduce the discrete cost functional

$$
\mathcal{J}_{\delta}^{h}(Q, f, g):=\int_{\Omega} Q \nabla\left(\mathcal{U}_{Q, f, g}^{h}-\Pi^{h} z_{\delta}\right) \cdot \nabla\left(\mathcal{U}_{Q, f, g}^{h}-\Pi^{h} z_{\delta}\right)
$$

where $(Q, f, g) \in \mathcal{H}_{a d}$.

Lemma 2.3. Assume that the sequence $\left(\Gamma_{n}\right)_{n}:=\left(Q_{n}, f_{n}, g_{n}\right)_{n} \subset \mathcal{H}_{\text {ad }}$ weakly converges to $\Gamma:=(Q, f, g)$ in $\mathbf{L}_{\text {sym }}^{2}(\Omega) \times L^{2}(\Omega) \times L^{2}(\partial \Omega)$. Then for any fixed $h>0$ the sequence $\left(\mathcal{U}_{\Gamma_{n}}^{h}\right)_{n} \subset \mathcal{V}_{1}^{h}$ converges to $\mathcal{U}_{\Gamma}^{h}$ in the $H^{1}(\Omega)$-norm.

Proof. Due to Remark 2.1 $\left(Q_{n}\right)_{n}$ has a subsequence denoted by the same symbol which is weakly* convergent in $\mathbf{L}_{\mathrm{sym}}^{\infty}(\Omega)$ to $Q$. Furthermore, by $\sqrt{2.5}$, the corresponding state sequence $\left(\mathcal{U}_{\Gamma_{n}}^{h}\right)_{n}$ is bounded in the finite dimensional space $\mathcal{V}_{1}^{h}$. A subsequence which is not relabelled and an element $\Theta^{h} \in \mathcal{V}_{1}^{h}$ then exist such that $\left(\mathcal{U}_{\Gamma_{n}}^{h}\right)_{n}$ converges to $\Theta^{h}$ in the $H^{1}(\Omega)$-norm. It follows from the equation 2.4 that

$$
\int_{\Omega} Q_{n} \nabla\left(\mathcal{U}_{\Gamma_{n}}^{h}-\mathcal{U}_{\Gamma}^{h}\right) \cdot \nabla \varphi^{h}=\int_{\Omega}\left(Q-Q_{n}\right) \nabla \mathcal{U}_{\Gamma}^{h} \cdot \nabla \varphi^{h}+\left(f_{n}-f, \varphi^{h}\right)+\left\langle g_{n}-g, \gamma \varphi^{h}\right\rangle
$$

for all $\varphi^{h} \in \mathcal{V}_{1}^{h}$. Taking $\varphi^{h}=\mathcal{U}_{\Gamma_{n}}^{h}-\mathcal{U}_{\Gamma}^{h}$, by 1.5 , we obtain that

$$
\begin{aligned}
\frac{C_{\Omega} \underline{q}}{1+C_{\Omega}}\left\|\mathcal{U}_{\Gamma_{n}}^{h}-\mathcal{U}_{\Gamma}^{h}\right\|_{H^{1}(\Omega)}^{2} \leq & \int_{\Omega}\left(Q-Q_{n}\right) \nabla \mathcal{U}_{\Gamma}^{h} \cdot \nabla\left(\mathcal{U}_{\Gamma_{n}}^{h}-\Theta^{h}+\Theta^{h}-\mathcal{U}_{\Gamma}^{h}\right) \\
& +\left(f_{n}-f, \mathcal{U}_{\Gamma_{n}}^{h}-\Theta^{h}+\Theta^{h}-\mathcal{U}_{\Gamma}^{h}\right)+\left\langle g_{n}-g, \gamma\left(\mathcal{U}_{\Gamma_{n}}^{h}-\Theta^{h}+\Theta^{h}-\mathcal{U}_{\Gamma}^{h}\right)\right\rangle \\
\leq & C\left\|\mathcal{U}_{\Gamma_{n}}^{h}-\Theta^{h}\right\|_{H^{1}(\Omega)}+\int_{\Omega}\left(Q-Q_{n}\right) \nabla \mathcal{U}_{\Gamma}^{h} \cdot \nabla\left(\Theta^{h}-\mathcal{U}_{\Gamma}^{h}\right) \\
& +\left(f_{n}-f, \Theta^{h}-\mathcal{U}_{\Gamma}^{h}\right)+\left\langle g_{n}-g, \gamma\left(\Theta^{h}-\mathcal{U}_{\Gamma}^{h}\right)\right\rangle .
\end{aligned}
$$

Since $Q_{n} \rightarrow Q$ weakly* in $\mathbf{L}_{\text {sym }}^{\infty}(\Omega)$, we get $\lim _{n \rightarrow \infty} \int_{\Omega}\left(Q-Q_{n}\right) \nabla \mathcal{U}_{\Gamma}^{h} \cdot \nabla\left(\Theta^{h}-\mathcal{U}_{\Gamma}^{h}\right)=0$. Sending $n$ to $\infty$, we thus obtain from the last inequality that $\lim _{n \rightarrow \infty}\left\|\mathcal{U}_{\Gamma_{n}}^{h}-\mathcal{U}_{\Gamma}^{h}\right\|_{H^{1}(\Omega)}=0$, which finishes the proof.

We now state the following useful result on the convexity of the cost functional.

Lemma 2.4. $\mathcal{J}_{\delta}^{h}$ is convex and continuous on $\mathcal{H}_{\text {ad }}$ with respect to the $\mathbf{L}_{\text {sym }}^{2}(\Omega) \times L^{2}(\Omega) \times L^{2}(\partial \Omega)$-norm.

Proof. The continuity of $\mathcal{J}_{\delta}^{h}$ follows directly from Lemma 2.3. We show that $\mathcal{J}_{\delta}^{h}$ is convex.

Let $\Gamma:=(Q, f, g) \in \mathcal{H}_{a d}$ and $\lambda:=(H, l, s) \in \mathbf{L}_{\mathrm{sym}}^{\infty}(\Omega) \times L^{2}(\Omega) \times L^{2}(\partial \Omega)$. We have that

$$
\mathcal{U}_{\Gamma}^{h^{\prime}}(\lambda)=\frac{\partial \mathcal{U}_{\Gamma}^{h}}{\partial Q} H+\frac{\partial \mathcal{U}_{\Gamma}^{h}}{\partial f} l+\frac{\partial \mathcal{U}_{\Gamma}^{h}}{\partial g} s \quad \text { and } \quad \mathcal{J}_{\delta}^{h^{\prime}}(\Gamma)(\lambda)=\frac{\partial \mathcal{J}_{\delta}^{h}(\Gamma)}{\partial Q} H+\frac{\partial \mathcal{J}_{\delta}^{h}(\Gamma)}{\partial f} l+\frac{\partial \mathcal{J}_{\delta}^{h}(\Gamma)}{\partial g} s .
$$

We compute for each term in the right hand side of the last equation. First we get

$$
\frac{\partial \mathcal{J}_{\delta}^{h}(\Gamma)}{\partial Q} H=\int_{\Omega} H \nabla\left(\mathcal{U}_{\Gamma}^{h}-\Pi^{h} z_{\delta}\right) \cdot \nabla\left(\mathcal{U}_{\Gamma}^{h}-\Pi^{h} z_{\delta}\right)+2 \int_{\Omega} Q \nabla\left(\frac{\partial \mathcal{U}_{\Gamma}^{h}}{\partial Q} H\right) \cdot \nabla\left(\mathcal{U}_{\Gamma}^{h}-\Pi^{h} z_{\delta}\right) .
$$


For the second term we have

$$
\frac{\partial \mathcal{J}_{\delta}^{h}(\Gamma)}{\partial f} l=2 \int_{\Omega} Q \nabla\left(\frac{\partial \mathcal{U}_{\Gamma}^{h}}{\partial f} l\right) \cdot \nabla\left(\mathcal{U}_{\Gamma}^{h}-\Pi^{h} z_{\delta}\right)
$$

Finally, we have for the third term

$$
\frac{\partial \mathcal{J}_{\delta}^{h}(\Gamma)}{\partial g} s=2 \int_{\Omega} Q \nabla\left(\frac{\partial \mathcal{U}_{\Gamma}^{h}}{\partial g} s\right) \cdot \nabla\left(\mathcal{U}_{\Gamma}^{h}-\Pi^{h} z_{\delta}\right) .
$$

Therefore,

$$
\begin{aligned}
\mathcal{J}_{\delta}^{h^{\prime}}(\Gamma)(\lambda) & =2 \int_{\Omega} Q \nabla\left(\frac{\partial \mathcal{U}_{\Gamma}^{h}}{\partial Q} H+\frac{\partial \mathcal{U}_{\Gamma}^{h}}{\partial f} l+\frac{\partial \mathcal{U}_{\Gamma}^{h}}{\partial g} s\right) \cdot \nabla\left(\mathcal{U}_{\Gamma}^{h}-\Pi^{h} z_{\delta}\right)+\int_{\Omega} H \nabla\left(\mathcal{U}_{\Gamma}^{h}-\Pi^{h} z_{\delta}\right) \cdot \nabla\left(\mathcal{U}_{\Gamma}^{h}-\Pi^{h} z_{\delta}\right) \\
& =2 \int_{\Omega} Q \nabla \mathcal{U}_{\Gamma}^{h^{\prime}}(\lambda) \cdot \nabla\left(\mathcal{U}_{\Gamma}^{h}-\Pi^{h} z_{\delta}\right)+\int_{\Omega} H \nabla\left(\mathcal{U}_{\Gamma}^{h}-\Pi^{h} z_{\delta}\right) \cdot \nabla\left(\mathcal{U}_{\Gamma}^{h}-\Pi^{h} z_{\delta}\right) \\
& =2 \int_{\Omega} Q \nabla \mathcal{U}_{\Gamma}^{h^{\prime}}(\lambda) \cdot \nabla\left(\mathcal{U}_{\Gamma}^{h}-\bar{\Pi}^{h} z_{\delta}\right)+\int_{\Omega} H \nabla\left(\mathcal{U}_{\Gamma}^{h}-\bar{\Pi}^{h} z_{\delta}\right) \cdot \nabla\left(\mathcal{U}_{\Gamma}^{h}-\bar{\Pi}^{h} z_{\delta}\right),
\end{aligned}
$$

where

$$
\bar{\Pi}^{h} z_{\delta}:=\Pi^{h} z_{\delta}-|\Omega|^{-1}\left\langle 1, \gamma \Pi^{h} z_{\delta}\right\rangle \in \mathcal{V}_{1}^{h} \quad \text { with } \nabla \bar{\Pi}^{h} z_{\delta}=\nabla \Pi^{h} z_{\delta} .
$$

By 2.6, we infer that

$$
\begin{aligned}
\mathcal{J}_{\delta}^{h^{\prime}}(\Gamma)(\lambda)= & -2 \int_{\Omega} H \nabla \mathcal{U}_{\Gamma}^{h} \cdot \nabla\left(\mathcal{U}_{\Gamma}^{h}-\bar{\Pi}^{h} z_{\delta}\right)+2\left(l, \mathcal{U}_{\Gamma}^{h}-\bar{\Pi}^{h} z_{\delta}\right)+2\left\langle s, \gamma\left(\mathcal{U}_{\Gamma}^{h}-\bar{\Pi}^{h} z_{\delta}\right)\right\rangle \\
& +\int_{\Omega} H \nabla\left(\mathcal{U}_{\Gamma}^{h}-\bar{\Pi}^{h} z_{\delta}\right) \cdot \nabla\left(\mathcal{U}_{\Gamma}^{h}-\bar{\Pi}^{h} z_{\delta}\right) \\
= & -\int_{\Omega} H \nabla \mathcal{U}_{\Gamma}^{h} \cdot \nabla \mathcal{U}_{\Gamma}^{h}+\int_{\Omega} H \nabla \bar{\Pi}^{h} z_{\delta} \cdot \nabla \bar{\Pi}^{h} z_{\delta}+2\left(l, \mathcal{U}_{\Gamma}^{h}-\bar{\Pi}^{h} z_{\delta}\right)+2\left\langle s, \gamma\left(\mathcal{U}_{\Gamma}^{h}-\bar{\Pi}^{h} z_{\delta}\right)\right\rangle .
\end{aligned}
$$

Therefore, by 2.6 again, we arrive at

$$
\begin{aligned}
\mathcal{J}_{\delta}^{h^{\prime \prime}}(\Gamma)(\lambda, \lambda) & =-2 \int_{\Omega} H \nabla \mathcal{U}_{\Gamma}^{h} \cdot \nabla \mathcal{U}_{\Gamma}^{h^{\prime}}(\lambda)+2\left(l, \mathcal{U}_{\Gamma}^{h^{\prime}}(\lambda)\right)+2\left\langle s, \gamma \mathcal{U}_{\Gamma}^{h^{\prime}}(\lambda)\right\rangle \\
& =2 \int_{\Omega} Q \nabla \mathcal{U}_{\Gamma}^{h^{\prime}}(\lambda) \cdot \nabla \mathcal{U}_{\Gamma}^{h^{\prime}}(\lambda) \geq 2 \frac{C_{\Omega} \underline{q}}{1+C_{\Omega}}\left\|\mathcal{U}_{\Gamma}^{h^{\prime}}(\lambda)\right\|_{H^{1}(\Omega)}^{2} \geq 0
\end{aligned}
$$

by 1.5 , which completes the proof.

Now we are in position to prove the main result of this section.

Theorem 2.5. The strictly convex minimization problem

$$
\min _{(Q, f, g) \in \mathcal{H}_{a d}} \Upsilon_{\delta}^{\rho, h}(Q, f, g):=\mathcal{J}_{\delta}^{h}(Q, f, g)+\rho \mathcal{R}(Q, f, g)
$$

attains a unique minimizer. Furthermore, an element $\Gamma:=(Q, f, g) \in \mathcal{H}_{\text {ad }}$ is the unique minimizer to $\left(\mathcal{P}_{\delta}^{\rho, h}\right)$ if and only if the system

$$
\begin{aligned}
Q(x) & =P_{\mathcal{K}}\left(\frac{1}{2 \rho}\left(\nabla \mathcal{U}_{\Gamma}^{h}(x) \otimes \nabla \mathcal{U}_{\Gamma}^{h}(x)-\nabla \bar{\Pi}^{h} z_{\delta}(x) \otimes \nabla \bar{\Pi}^{h} z_{\delta}(x)\right)\right), \\
f(x) & =\frac{1}{\rho}\left(\bar{\Pi}^{h} z_{\delta}(x)-\mathcal{U}_{\Gamma}^{h}(x)\right), \\
g(x) & =\frac{1}{\rho} \gamma\left(\bar{\Pi}^{h} z_{\delta}(x)-\mathcal{U}_{\Gamma}^{h}(x)\right)
\end{aligned}
$$

holds for a.e. in $\Omega$, where $\bar{\Pi}^{h}$ was generated from $\Pi^{h}$ according to (2.13). 
Proof. Let $\left(\Gamma_{n}\right)_{n}:=\left(Q_{n}, f_{n}, g_{n}\right)_{n} \subset \mathcal{H}_{a d}$ be a minimizing sequence of $\left(\mathcal{P}_{\delta}^{\rho, h}\right)$, i.e.

$$
\lim _{n \rightarrow \infty} \Upsilon_{\delta}^{\rho, h}\left(\Gamma_{n}\right)=\inf _{(Q, f, g) \in \mathcal{H}_{a d}} \Upsilon_{\delta}^{\rho, h}(Q, f, g)
$$

The sequence $\left(\Gamma_{n}\right)_{n}$ is thus bounded in the $\mathbf{L}_{\text {sym }}^{2}(\Omega) \times L^{2}(\Omega) \times L^{2}(\partial \Omega)$-norm. A subsequence not relabelled and an element $\Gamma:=(Q, f, g) \in \mathbf{L}_{\text {sym }}^{2}(\Omega) \times L^{2}(\Omega) \times L^{2}(\partial \Omega)$ exist such that $\Gamma_{n} \rightarrow \Gamma$ weakly in $\mathbf{L}_{\text {sym }}^{2}(\Omega) \times$ $L^{2}(\Omega) \times L^{2}(\partial \Omega)$. On the other hand, since $\mathcal{H}_{a d}$ is a convex, closed subset of $\mathbf{L}_{\mathrm{sym}}^{2}(\Omega) \times L^{2}(\Omega) \times L^{2}(\partial \Omega)$, so is weakly closed, it follows that $\Gamma \in \mathcal{H}_{a d}$. By Lemma 2.4. $\mathcal{J}_{\delta}^{h}$ and $\mathcal{R}$ are both weakly lower semi-continuous on $\mathcal{H}_{a d}$ which yields that

$$
\mathcal{J}_{\delta}^{h}(\Gamma) \leq \liminf _{n \rightarrow \infty} \mathcal{J}_{\delta}^{h}\left(\Gamma_{n}\right) \text { and } \mathcal{R}(\Gamma) \leq \liminf _{n \rightarrow \infty} \mathcal{R}\left(\Gamma_{n}\right)
$$

We therefore have that

$$
\begin{aligned}
\mathcal{J}_{\delta}^{h}(\Gamma)+\mathcal{R}(\Gamma) & \leq \liminf _{n \rightarrow \infty} \mathcal{J}_{\delta}^{h}\left(\Gamma_{n}\right)+\liminf _{n \rightarrow \infty} \mathcal{R}\left(\Gamma_{n}\right) \leq \liminf _{n \rightarrow \infty}\left(\mathcal{J}_{\delta}^{h}\left(\Gamma_{n}\right)+\mathcal{R}\left(\Gamma_{n}\right)\right) \\
& =\lim _{n \rightarrow \infty} \Upsilon_{\delta}^{\rho, h}\left(\Gamma_{n}\right)=\inf _{(Q, f, g) \in \mathcal{H}_{a d}} \Upsilon_{\delta}^{\rho, h}(Q, f, g),
\end{aligned}
$$

and $\Gamma$ is then a minimizer to $\left(\mathcal{P}_{\delta}^{\rho, h}\right)$. Since $\Upsilon_{\delta}^{\rho, h}$ is strictly convex, this minimizer is unique. Next, an element $\Gamma:=(Q, f, g) \in \mathcal{H}_{a d}$ is the minimizer to $\left(\mathcal{P}_{\delta}^{\rho, h}\right)$ if and only if $\Upsilon_{\delta}^{\rho, h^{\prime}}(\Gamma)(\bar{\Gamma}-\Gamma) \geq 0$ for all $\bar{\Gamma}=(H, l, s) \in \mathcal{H}_{a d}$. Then, in view of 2.14, we get that

$$
\begin{aligned}
0 \leq & \int_{\Omega}(H-Q) \nabla \bar{\Pi}^{h} z_{\delta} \cdot \nabla \bar{\Pi}^{h} z_{\delta}-\int_{\Omega}(H-Q) \nabla \mathcal{U}_{\Gamma}^{h} \cdot \nabla \mathcal{U}_{\Gamma}^{h}+2 \rho(H-Q, Q) \\
& +2\left(l-f, \mathcal{U}_{\Gamma}^{h}-\bar{\Pi}^{h} z_{\delta}\right)+2 \rho(l-f, f)+2\left\langle s-g, \gamma\left(\mathcal{U}_{\Gamma}^{h}-\bar{\Pi}^{h} z_{\delta}\right)\right\rangle+2 \rho\langle s-g, g\rangle \\
= & \int_{\Omega}(H-Q) \cdot\left(\nabla \bar{\Pi}^{h} z_{\delta} \otimes \nabla \bar{\Pi}^{h} z_{\delta}-\nabla \mathcal{U}_{\Gamma}^{h} \otimes \nabla \mathcal{U}_{\Gamma}^{h}+2 \rho Q\right) \\
& +2\left(l-f, \mathcal{U}_{\Gamma}^{h}-\bar{\Pi}^{h} z_{\delta}+\rho f\right)+2\left\langle s-g, \gamma\left(\mathcal{U}_{\Gamma}^{h}-\bar{\Pi}^{h} z_{\delta}\right)+\rho g\right\rangle
\end{aligned}
$$

for all $\bar{\Gamma}=(H, l, s) \in \mathcal{H}_{a d}$. Taking $\bar{\Gamma}_{1}=(H, f, g), \bar{\Gamma}_{2}=(Q, l, g)$ and $\bar{\Gamma}_{3}=(Q, f, s)$ into the above inequality we obtain the system 2.15-2.17). The proof is completed.

Remark 2.6. We denote by

$$
\begin{aligned}
& \mathcal{V}_{0}^{h}:=\left\{\varphi^{h} \in L^{2}(\Omega) \quad \mid \quad \varphi^{h}=\text { const for all triangulations } T \in \mathcal{T}^{h}\right\}, \\
& \mathcal{E}_{1}^{h}:=\left\{\varphi^{h} \in C(\partial \Omega) \quad \mid \varphi_{\mid e}^{h} \in \mathcal{P}_{1} \text { for all boundary edges } e \text { of } \mathcal{T}^{h}\right\} .
\end{aligned}
$$

Since $\mathcal{U}_{\Gamma}^{h} \in \mathcal{V}_{1}^{h}$ and $\bar{\Pi}^{h} z_{\delta} \in \mathcal{V}_{1}^{h}$, the system $2.15-2.17$ shows that every solution of $\left(\mathcal{P}_{\delta}^{\rho, h}\right)$ automatically belongs to the finite dimensional space $\mathcal{V}_{0}^{h{ }^{d \times d}} \times \mathcal{V}_{1}^{h} \times \mathcal{E}_{1}^{h}$.

\section{Convergence}

For abbreviation in what follows we denote by $C$ a generic positive constant independent of the mesh size $h$, the noise level $\delta$ and the regularization parameter $\rho$. By $(2.8)$ and $(2.9)$, we can introduce for each $\Phi \in H^{1}(\Omega)$

$$
\chi_{\Phi}^{h}:=\left\|\Phi-\Pi^{h} \Phi\right\|_{H^{1}(\Omega)} \quad \text { which satisfies } \quad \lim _{h \rightarrow 0} \chi_{\Phi}^{h}=0 \text { and } 0 \leq \chi_{\Phi}^{h} \leq C h
$$

in case $\Phi \in H^{2}(\Omega)$. Likewise, by 2.7 , for all $\Gamma \in \mathcal{H}_{a d}$

$$
\beta_{\mathcal{U}_{\Gamma}}^{h}:=\left\|\mathcal{U}_{\Gamma}-\mathcal{U}_{\Gamma}^{h}\right\|_{H^{1}(\Omega)} \rightarrow 0 \text { as } h \rightarrow 0 \text { and } 0 \leq \beta_{\mathcal{U}_{\Gamma}}^{h} \leq C h \text { as } \mathcal{U}_{\Gamma} \in H^{2}(\Omega) .
$$

Furthermore, by (2.9), we get

$$
\left\|\Pi^{h}\right\|_{\mathcal{L}\left(L^{2}(\Omega), L^{2}(\Omega)\right)} \leq C \text { and }\left\|\Pi^{h}\right\|_{\mathcal{L}\left(H^{1}(\Omega), H^{1}(\Omega)\right)} \leq C .
$$


Thus, it follows from the inverse inequality (cf. [6, 12]):

$$
\left\|\varphi^{h}\right\|_{H^{1}(\Omega)} \leq C h^{-1}\left\|\varphi^{h}\right\|_{L^{2}(\Omega)} \quad \text { for all } \quad \varphi^{h} \in\left\{\varphi^{h} \in C(\bar{\Omega}) \quad \mid \quad \varphi_{\mid T}^{h} \in \mathcal{P}_{1}(T) \quad \text { for all } \quad T \in \mathcal{T}^{h}\right\}
$$

that

$$
\begin{aligned}
\left\|\Phi^{\dagger}-\Pi^{h} z_{\delta}\right\|_{H^{1}(\Omega)} & \leq\left\|\Pi^{h}\left(\Phi^{\dagger}-z_{\delta}\right)\right\|_{H^{1}(\Omega)}+\left\|\Phi^{\dagger}-\Pi^{h} \Phi^{\dagger}\right\|_{H^{1}(\Omega)} \leq C h^{-1}\left\|\Pi^{h}\left(\Phi^{\dagger}-z_{\delta}\right)\right\|_{L^{2}(\Omega)}+\chi_{\Phi^{\dagger}}^{h} \\
& \leq C h^{-1}\left\|\Pi^{h}\right\|_{\mathcal{L}\left(L^{2}(\Omega), L^{2}(\Omega)\right)}\left\|\Phi^{\dagger}-z_{\delta}\right\|_{L^{2}(\Omega)}+\chi_{\Phi^{\dagger}}^{h} \leq C h^{-1} \delta+\chi_{\Phi^{\dagger}}^{h} .
\end{aligned}
$$

The following result shows the convergence of finite element approximations to the unique minimum norm solution $\Gamma^{\dagger}:=\left(Q^{\dagger}, f^{\dagger}, g^{\dagger}\right)$ of the identification problem, which is defined by 1.8$)$.

Theorem 3.1. Let $\left(h_{n}\right)_{n}$ be a sequence with $\lim _{n \rightarrow \infty} h_{n}=0$ and $\left(\delta_{n}\right)_{n}$ and $\left(\rho_{n}\right)_{n}$ are any positive sequences such that

$$
\rho_{n} \rightarrow 0, \quad \frac{\delta_{n}}{h_{n} \sqrt{\rho_{n}}} \rightarrow 0, \quad \frac{\beta_{\mathcal{U}^{\dagger}}^{h_{n}}}{\sqrt{\rho_{n}}} \rightarrow 0 \quad \text { and } \quad \frac{\chi_{\Phi^{\dagger}}^{h_{n}}}{\sqrt{\rho_{n}}} \rightarrow 0 \quad \text { as } \quad n \rightarrow \infty
$$

Assume that $\left(z_{\delta_{n}}\right)_{n} \subset L^{2}(\Omega)$ is a sequence satisfying $\left\|z_{\delta_{n}}-\Phi^{\dagger}\right\|_{L^{2}(\Omega)} \leq \delta_{n}$ and $\Gamma_{n}:=\left(Q_{n}, f_{n}, g_{n}\right)$ is the unique minimizer of the problem $\left(\mathcal{P}_{\delta_{n}}^{\rho_{n}, h_{n}}\right)$ for each $n \in N$. Then the sequence $\left(\Gamma_{n}\right)_{n}$ converges to $\Gamma^{\dagger}$ in the $\mathbf{L}_{\text {sym }}^{2}(\Omega) \times L^{2}(\Omega) \times L^{2}(\partial \Omega)$-norm as $n \rightarrow \infty$. Furthermore, the corresponding discrete state sequence $\left(\mathcal{U}_{\Gamma_{n}}^{h_{n}}\right)_{n}$ also converges to $\Phi^{\dagger}$ in the $H^{1}(\Omega)$-norm.

Remark 3.2. In case $\Phi^{\dagger}=\mathcal{U}_{\Gamma^{\dagger}} \in H^{2}(\Omega)$ we have $0 \leq \beta_{\mathcal{U}_{\Gamma^{\dagger}}}^{h_{n}}, \chi_{\Phi^{\dagger}}^{h_{n}} \leq C h_{n}$. Therefore, the convergence of Theorem 3.1 is obtained if $\delta_{n} \sim h_{n}^{2}$ and the sequence $\left(\rho_{n}\right)_{n}$ is chosen such that

$$
\rho_{n} \rightarrow 0 \quad \text { and } \quad \frac{h_{n}}{\sqrt{\rho_{n}}} \rightarrow 0 \quad \text { as } n \rightarrow \infty \text {. }
$$

To prove Theorem 3.1, we need the following auxiliary estimate.

Lemma 3.3. There holds the estimate

$$
\mathcal{J}_{\delta}^{h}\left(\Gamma^{\dagger}\right) \leq C\left(h^{-2} \delta^{2}+\left(\chi_{\Phi^{\dagger}}^{h}\right)^{2}+\left(\beta_{\mathcal{U}_{\Gamma^{\dagger}}}^{h}\right)^{2}\right) .
$$

Proof. We have with $\Phi^{\dagger}=\mathcal{U}_{\Gamma^{\dagger}}$ and (3.2) that

$$
\begin{aligned}
\mathcal{J}_{\delta}^{h}\left(\Gamma^{\dagger}\right) & =\int_{\Omega} Q^{\dagger} \nabla\left(\mathcal{U}_{\Gamma^{\dagger}}^{h}-\Pi^{h} z_{\delta}\right) \cdot \nabla\left(\mathcal{U}_{\Gamma^{\dagger}}^{h}-\Pi^{h} z_{\delta}\right) \leq \bar{q}\left\|\mathcal{U}_{\Gamma^{\dagger}}^{h}-\Pi^{h} z_{\delta}\right\|_{H^{1}(\Omega)}^{2} \\
& =\bar{q}\left\|\mathcal{U}_{\Gamma^{\dagger}}^{h}-\mathcal{U}_{\Gamma^{\dagger}}+\Phi^{\dagger}-\Pi^{h} z_{\delta}\right\|_{H^{1}(\Omega)}^{2} \leq C\left(\left\|\mathcal{U}_{\Gamma^{\dagger}}^{h}-\mathcal{U}_{\Gamma^{\dagger}}\right\|_{H^{1}(\Omega)}^{2}+\left\|\Phi^{\dagger}-\Pi^{h} z_{\delta}\right\|_{H^{1}(\Omega)}^{2}\right) \\
& \leq C\left(h^{-2} \delta^{2}+\left(\chi_{\Phi^{\dagger}}^{h}\right)^{2}+\left(\beta_{\mathcal{U}_{\Gamma^{\dagger}}}^{h}\right)^{2}\right),
\end{aligned}
$$

which finishes the proof.

Proof of Theorem 3.1. By the optimality of $\Gamma_{n}$ and Lemma 3.3 we have that

$$
\begin{aligned}
\mathcal{J}_{\delta_{n}}^{h_{n}}\left(\Gamma_{n}\right)+\rho_{n} \mathcal{R}\left(\Gamma_{n}\right) & \leq \mathcal{J}_{\delta_{n}}^{h_{n}}\left(\Gamma^{\dagger}\right)+\rho_{n} \mathcal{R}\left(\Gamma^{\dagger}\right) \\
& \leq C\left(h_{n}^{-2} \delta_{n}^{2}+\left(\chi_{\Phi^{\dagger}}^{h_{n}}\right)^{2}+\left(\beta_{\mathcal{U}^{\dagger}}^{h_{n}}\right)^{2}\right)+\rho_{n} \mathcal{R}\left(\Gamma^{\dagger}\right)
\end{aligned}
$$

which yields

$$
\lim _{n \rightarrow \infty} \mathcal{J}_{\delta_{n}}^{h_{n}}\left(\Gamma_{n}\right)=0
$$

and

$$
\limsup _{n \rightarrow \infty} \mathcal{R}\left(\Gamma_{n}\right) \leq \mathcal{R}\left(\Gamma^{\dagger}\right)
$$


A subsequence of the sequence $\left(\Gamma_{n}\right)_{n}$ denoted by the same symbol and an element $\Gamma_{0}:=\left(Q_{0}, f_{0}, g_{0}\right) \in \mathcal{H}_{a d}$ then exist such that

$$
\begin{aligned}
Q_{n} & \rightarrow Q_{0} \quad \text { weakly* in } \quad \mathbf{L}_{\text {sym }}^{\infty}(\Omega), \\
f_{n} & \rightarrow f_{0} \text { weakly in } L^{2}(\Omega), \\
g_{n} & \rightarrow g_{0} \text { weakly in } L^{2}(\partial \Omega) .
\end{aligned}
$$

We will show that $\left(\Gamma_{n}\right)_{n}$ converges to $\Gamma_{0}$ in the $\mathbf{L}_{\text {sym }}^{2}(\Omega) \times L^{2}(\Omega) \times L^{2}(\partial \Omega)$-norm and $\Gamma_{0}=\Gamma^{\dagger}$. We have from (3.2) that

$$
\lim _{n \rightarrow \infty}\left\|\Pi^{h_{n}} z_{\delta_{n}}-\mathcal{U}_{\Gamma^{\dagger}}\right\|_{H^{1}(\Omega)} \leq \lim _{n \rightarrow \infty}\left(C h_{n}^{-1} \delta_{n}+\chi_{\Phi^{\dagger}}^{h_{n}}\right)=0 .
$$

Combining this with $\lim _{n \rightarrow \infty}\left\|\mathcal{U}_{\Gamma_{0}}-\mathcal{U}_{\Gamma_{0}}^{h_{n}}\right\|_{H^{1}(\Omega)}=0$ from 2.7 , we arrive at

$$
\begin{aligned}
\lim _{n \rightarrow \infty} \mathcal{J}_{\delta_{n}}^{h_{n}}\left(\Gamma_{0}\right) & =\lim _{n \rightarrow \infty} \int_{\Omega} Q_{0} \nabla\left(\mathcal{U}_{\Gamma_{0}}^{h_{n}}-\Pi^{h_{n}} z_{\delta_{n}}\right) \cdot \nabla\left(\mathcal{U}_{\Gamma_{0}}^{h_{n}}-\Pi^{h_{n}} z_{\delta_{n}}\right) \\
& =\int_{\Omega} Q_{0} \nabla\left(\mathcal{U}_{\Gamma_{0}}-\mathcal{U}_{\Gamma^{\dagger}}\right) \cdot \nabla\left(\mathcal{U}_{\Gamma_{0}}-\mathcal{U}_{\Gamma^{\dagger}}\right) .
\end{aligned}
$$

Now for each fixed $n$ we consider an arbitrary subsequence $\left(\Gamma_{n_{m}}\right)_{m}$ of $\left(\Gamma_{n}\right)_{n}$. By the weakly l.s.c. property of the functional $\mathcal{J}_{\delta_{n}}^{h_{n}}$ (cf. Lemma 2.4, we obtain that

$$
\mathcal{J}_{\delta_{n}}^{h_{n}}\left(\Gamma_{0}\right) \leq \liminf _{m \rightarrow \infty} \mathcal{J}_{\delta_{n}}^{h_{n}}\left(\Gamma_{n_{m}}\right)
$$

Again, using the convexity of $\mathcal{J}_{\delta_{n}}^{h_{n}}$, we get that

$$
\mathcal{J}_{\delta_{n}}^{h_{n}}\left(\Gamma_{n}\right) \geq \mathcal{J}_{\delta_{n}}^{h_{n}}\left(\Gamma_{n_{m}}\right)+\mathcal{J}_{\delta_{n}}^{h_{n}{ }^{\prime}}\left(\Gamma_{n_{m}}\right)\left(\Gamma_{n}-\Gamma_{n_{m}}\right) .
$$

By 1.5 , we thus arrive at

$$
\begin{aligned}
C\left\|\mathcal{U}_{\Gamma_{0}}-\mathcal{U}_{\Gamma^{\dagger}}\right\|_{H^{1}(\Omega)}^{2} & \leq \int_{\Omega} Q_{0} \nabla\left(\mathcal{U}_{\Gamma_{0}}-\mathcal{U}_{\Gamma^{\dagger}}\right) \cdot \nabla\left(\mathcal{U}_{\Gamma_{0}}-\mathcal{U}_{\Gamma^{\dagger}}\right) \\
& =\lim _{n \rightarrow \infty} \mathcal{J}_{\delta_{n}}^{h_{n}}\left(\Gamma_{0}\right) \leq \lim _{n \rightarrow \infty}\left(\liminf _{m \rightarrow \infty} \mathcal{J}_{\delta_{n}}^{h_{n}}\left(\Gamma_{n_{m}}\right)\right) \\
& \leq \lim _{n \rightarrow \infty} \liminf _{m \rightarrow \infty}\left(\mathcal{J}_{\delta_{n}}^{h_{n}}\left(\Gamma_{n}\right)+\mathcal{J}_{\delta_{n}}^{h_{n}{ }^{\prime}}\left(\Gamma_{n_{m}}\right)\left(\Gamma_{n_{m}}-\Gamma_{n}\right)\right) .
\end{aligned}
$$

Using (3.4), we infer from the last inequality that

$$
C\left\|\mathcal{U}_{\Gamma_{0}}-\mathcal{U}_{\Gamma^{\dagger}}\right\|_{H^{1}(\Omega)}^{2} \leq \lim _{n \rightarrow \infty} \liminf _{m \rightarrow \infty} \mathcal{J}_{\delta_{n}{ }^{h_{n}}{ }^{\prime}}\left(\Gamma_{n_{m}}\right)\left(\Gamma_{n_{m}}-\Gamma_{n}\right) .
$$

In view of 2.14 we get that

$$
\begin{aligned}
\mathcal{J}_{\delta_{n}}^{h_{n}{ }^{\prime}}\left(\Gamma_{n_{m}}\right)\left(\Gamma_{n_{m}}-\Gamma_{n}\right)= & \int_{\Omega}\left(Q_{n_{m}}-Q_{n}\right) \nabla \bar{\Pi}^{h_{n}} z_{\delta_{n}} \cdot \nabla \bar{\Pi}^{h_{n}} z_{\delta_{n}} \\
& -2\left(f_{n_{m}}-f_{n}, \bar{\Pi}^{h_{n}} z_{\delta_{n}}\right)-2\left\langle g_{n_{m}}-g_{n}, \gamma \bar{\Pi}^{h_{n}} z_{\delta_{n}}\right\rangle \\
& -\int_{\Omega}\left(Q_{n_{m}}-Q_{n}\right) \nabla \mathcal{U}_{\Gamma_{n_{m}}}^{h_{n}} \cdot \nabla \mathcal{U}_{\Gamma_{n_{m}}}^{h_{n}}+2\left(f_{n_{m}}-f_{n}, \mathcal{U}_{\Gamma_{n_{m}}}^{h_{n}}\right)+2\left\langle g_{n_{m}}-g_{n}, \gamma \mathcal{U}_{\Gamma_{n_{m}}}^{h_{n}}\right\rangle \\
:= & A_{1}-2 A_{2}-2 A_{3}-A_{4}+2 A_{5}+2 A_{6} .
\end{aligned}
$$

Since $Q_{n_{m}} \rightarrow Q_{0}$ weakly* in $\mathbf{L}_{\text {sym }}^{\infty}(\Omega)$ as $m \rightarrow \infty$, we have for the first term that

$$
\begin{aligned}
\lim _{n \rightarrow \infty} \lim _{m \rightarrow \infty} A_{1}:= & \lim _{n \rightarrow \infty}\left(\lim _{m \rightarrow \infty} \int_{\Omega}\left(Q_{n_{m}}-Q_{n}\right) \nabla \bar{\Pi}^{h_{n}} z_{\delta_{n}} \cdot \nabla \bar{\Pi}^{h_{n}} z_{\delta_{n}}\right) \\
= & \lim _{n \rightarrow \infty} \int_{\Omega}\left(Q_{0}-Q_{n}\right) \nabla \bar{\Pi}^{h_{n}} z_{\delta_{n}} \cdot \nabla \bar{\Pi}^{h_{n}} z_{\delta_{n}} \\
= & \lim _{n \rightarrow \infty} \int_{\Omega}\left(Q_{0}-Q_{n}\right) \nabla \mathcal{U}_{\Gamma^{\dagger}} \cdot \nabla \mathcal{U}_{\Gamma^{\dagger}} \\
& +\lim _{n \rightarrow \infty} \int_{\Omega}\left(Q_{0}-Q_{n}\right) \nabla\left(\bar{\Pi}^{h_{n}} z_{\delta_{n}}-\mathcal{U}_{\Gamma^{\dagger}}\right) \cdot \nabla\left(\bar{\Pi}^{h_{n}} z_{\delta_{n}}+\mathcal{U}_{\Gamma^{\dagger}}\right) \\
= & \lim _{n \rightarrow \infty} \int_{\Omega}\left(Q_{0}-Q_{n}\right) \nabla\left(\bar{\Pi}^{h_{n}} z_{\delta_{n}}-\mathcal{U}_{\Gamma^{\dagger}}\right) \cdot \nabla\left(\bar{\Pi}^{h_{n}} z_{\delta_{n}}+\mathcal{U}_{\Gamma^{\dagger}}\right)
\end{aligned}
$$


since $\lim _{n \rightarrow \infty} \int_{\Omega}\left(Q_{0}-Q_{n}\right) \nabla \mathcal{U}_{\Gamma^{\dagger}} \cdot \nabla \mathcal{U}_{\Gamma^{\dagger}}=0$, due to $Q_{n} \rightarrow Q_{0}$ weakly* in $\mathbf{L}_{\text {sym }}^{\infty}(\Omega)$. Furthermore, by (3.6), we get that

$$
\begin{aligned}
& \lim _{n \rightarrow \infty}\left|\int_{\Omega}\left(Q_{0}-Q_{n}\right) \nabla\left(\bar{\Pi}^{h_{n}} z_{\delta_{n}}-\mathcal{U}_{\Gamma^{\dagger}}\right) \cdot \nabla\left(\bar{\Pi}^{h_{n}} z_{\delta_{n}}+\mathcal{U}_{\Gamma^{\dagger}}\right)\right| \\
& \quad \leq \lim _{n \rightarrow \infty} C\left\|\nabla\left(\bar{\Pi}^{h_{n}} z_{\delta_{n}}-\mathcal{U}_{\Gamma^{\dagger}}\right)\right\|_{L^{2}(\Omega)}=\lim _{n \rightarrow \infty} C\left\|\nabla\left(\Pi^{h_{n}} z_{\delta_{n}}-\mathcal{U}_{\Gamma^{\dagger}}\right)\right\|_{L^{2}(\Omega)} \\
& \quad \leq C \lim _{n \rightarrow \infty}\left\|\Pi^{h_{n}} z_{\delta_{n}}-\mathcal{U}_{\Gamma^{\dagger}}\right\|_{H^{1}(\Omega)}=0 .
\end{aligned}
$$

Therefore,

$$
\lim _{n \rightarrow \infty} \lim _{m \rightarrow \infty} A_{1}=0
$$

On the other hand, we get

$$
\begin{aligned}
\lim _{n \rightarrow \infty} \lim _{m \rightarrow \infty} A_{2} & :=\lim _{n \rightarrow \infty} \lim _{m \rightarrow \infty}\left(f_{n_{m}}-f_{n}, \bar{\Pi}^{h_{n}} z_{\delta_{n}}\right)=\lim _{n \rightarrow \infty}\left(f_{0}-f_{n}, \bar{\Pi}^{h_{n}} z_{\delta_{n}}\right) \\
& =\underbrace{\lim _{n \rightarrow \infty}\left(f_{0}-f_{n}, \mathcal{U}_{\Gamma^{\dagger}}\right)}_{=0}+\lim _{n \rightarrow \infty}\left(f_{0}-f_{n}, \bar{\Pi}^{h_{n}} z_{\delta_{n}}-\mathcal{U}_{\Gamma^{\dagger}}\right) \\
& \leq C \lim _{n \rightarrow \infty}\left\|\bar{\Pi}^{h_{n}} z_{\delta_{n}}-\mathcal{U}_{\Gamma^{\dagger}}\right\|_{L^{2}(\Omega)} \\
& \leq C \lim _{n \rightarrow \infty}\left\|\nabla\left(\bar{\Pi}^{h_{n}} z_{\delta_{n}}-\mathcal{U}_{\Gamma^{\dagger}}\right)\right\|_{L^{2}(\Omega)}=0 .
\end{aligned}
$$

We now have that

$$
\begin{aligned}
\lim _{n \rightarrow \infty} \lim _{m \rightarrow \infty} A_{3}:=\lim _{n \rightarrow \infty} \lim _{m \rightarrow \infty}\left\langle g_{n_{m}}-g_{n}, \gamma \bar{\Pi}^{h_{n}} z_{\delta_{n}}\right\rangle=\lim _{n \rightarrow \infty}\left\langle g_{0}-g_{n}, \gamma \bar{\Pi}^{h_{n}} z_{\delta_{n}}\right\rangle \\
=\lim _{n \rightarrow \infty}\left\langle g_{0}-g_{n}, \gamma \Pi^{h_{n}} z_{\delta_{n}}\right\rangle-|\partial \Omega|^{-1} \lim _{n \rightarrow \infty}\left\langle g_{0}-g_{n},\left\langle 1, \gamma \Pi^{h_{n}} z_{\delta_{n}}\right\rangle\right\rangle
\end{aligned}
$$

with

$$
\begin{aligned}
\lim _{n \rightarrow \infty} & \left\langle g_{0}-g_{n}, \gamma \Pi^{h_{n}} z_{\delta_{n}}\right\rangle \\
& =\lim _{n \rightarrow \infty}\left\langle g_{0}-g_{n}, \gamma\left(\Pi^{h_{n}} z_{\delta_{n}}-\mathcal{U}_{\Gamma^{\dagger}}\right)\right\rangle+\underbrace{\lim _{n \rightarrow \infty}\left\langle g_{0}-g_{n}, \gamma \mathcal{U}_{\Gamma^{\dagger}}\right\rangle}_{=0} \\
& \leq C \lim _{n \rightarrow \infty}\left\|g_{0}-g_{n}\right\|_{L^{2}(\partial \Omega)}\|\gamma\|_{\mathcal{L}\left(H^{1}(\Omega), H^{1 / 2}(\partial \Omega)\right)}\left\|\Pi^{h_{n}} z_{\delta_{n}}-\mathcal{U}_{\Gamma^{\dagger}}\right\|_{H^{1}(\Omega)} \\
& \leq C \lim _{n \rightarrow \infty}\left\|\Pi^{h_{n}} z_{\delta_{n}}-\mathcal{U}_{\Gamma^{\dagger}}\right\|_{H^{1}(\Omega)}=0
\end{aligned}
$$

and

$$
\begin{aligned}
\lim _{n \rightarrow \infty}\left\langle g_{0}-g_{n},\left\langle 1, \gamma \Pi^{h_{n}} z_{\delta_{n}}\right\rangle\right\rangle & \leq \lim _{n \rightarrow \infty}\left|\left\langle 1, \gamma \Pi^{h_{n}} z_{\delta_{n}}\right\rangle\right|\left|\left\langle g_{0}-g_{n}, 1\right\rangle\right| \\
& \leq C \lim _{n \rightarrow \infty}\left\|\Pi^{h_{n}} z_{\delta_{n}}\right\|_{H^{1}(\Omega)}\left|\left\langle g_{0}-g_{n}, 1\right\rangle\right| \\
& \leq C \lim _{n \rightarrow \infty}\left|\left\langle g_{0}-g_{n}, 1\right\rangle\right|=0
\end{aligned}
$$

so that

$$
\lim _{n \rightarrow \infty} \lim _{m \rightarrow \infty} A_{3}=0
$$

Next, we rewrite

$$
\begin{aligned}
\lim _{n \rightarrow \infty} \lim _{m \rightarrow \infty} A_{4}:= & \lim _{n \rightarrow \infty} \lim _{m \rightarrow \infty} \int_{\Omega}\left(Q_{n_{m}}-Q_{n}\right) \nabla \mathcal{U}_{\Gamma_{n_{m}}}^{h_{n}} \cdot \nabla \mathcal{U}_{\Gamma_{n_{m}}}^{h_{n}} \\
= & \lim _{n \rightarrow \infty} \lim _{m \rightarrow \infty} \int_{\Omega}\left(Q_{n_{m}}-Q_{n}\right) \nabla \mathcal{U}_{\Gamma_{0}}^{h_{n}} \cdot \nabla \mathcal{U}_{\Gamma_{0}}^{h_{n}} \\
& +\lim _{n \rightarrow \infty} \lim _{m \rightarrow \infty} \int_{\Omega}\left(Q_{n_{m}}-Q_{n}\right) \nabla\left(\mathcal{U}_{\Gamma_{n_{m}}}^{h_{n}}-\mathcal{U}_{\Gamma_{0}}^{h_{n}}\right) \cdot \nabla\left(\mathcal{U}_{\Gamma_{n_{m}}}^{h_{n}}+\mathcal{U}_{\Gamma_{0}}^{h_{n}}\right) .
\end{aligned}
$$


By (2.7), likewise as 3.9 , we get that

$$
\lim _{n \rightarrow \infty} \lim _{m \rightarrow \infty} \int_{\Omega}\left(Q_{n_{m}}-Q_{n}\right) \nabla \mathcal{U}_{\Gamma_{0}}^{h_{n}} \cdot \nabla \mathcal{U}_{\Gamma_{0}}^{h_{n}}=0 .
$$

Furthermore, we have

$$
\left|\int_{\Omega}\left(Q_{n_{m}}-Q_{n}\right) \nabla\left(\mathcal{U}_{\Gamma_{n_{m}}}^{h_{n}}-\mathcal{U}_{\Gamma_{0}}^{h_{n}}\right) \cdot \nabla\left(\mathcal{U}_{\Gamma_{n_{m}}}^{h_{n}}+\mathcal{U}_{\Gamma_{0}}^{h_{n}}\right)\right| \leq C\left\|\mathcal{U}_{\Gamma_{n_{m}}}^{h_{n}}-\mathcal{U}_{\Gamma_{0}}^{h_{n}}\right\|_{H^{1}(\Omega)} .
$$

By Lemma 2.3. for each fixed $n$ we have that the sequence $\left(\mathcal{U}_{\Gamma_{n_{m}}}^{h_{n}}\right)_{m} \subset \mathcal{V}_{1}^{h_{n}}$ converges to $\mathcal{U}_{\Gamma_{0}}^{h_{n}}$ in the $H^{1}(\Omega)$-norm as $m$ tends to $\infty$. Then we deduce that

$$
\begin{aligned}
& \lim _{n \rightarrow \infty} \lim _{m \rightarrow \infty}\left|\int_{\Omega}\left(Q_{n_{m}}-Q_{n}\right) \nabla\left(\mathcal{U}_{\Gamma_{n_{m}}}^{h_{n}}-\mathcal{U}_{\Gamma_{0}}^{h_{n}}\right) \cdot \nabla\left(\mathcal{U}_{\Gamma_{n_{m}}}^{h_{n}}+\mathcal{U}_{\Gamma_{0}}^{h_{n}}\right)\right| \\
& \quad \leq C \lim _{n \rightarrow \infty} \lim _{m \rightarrow \infty}\left\|\mathcal{U}_{\Gamma_{n_{m}}}^{h_{n}}-\mathcal{U}_{\Gamma_{0}}^{h_{n}}\right\|_{H^{1}(\Omega)}=C \lim _{n \rightarrow \infty}\left\|\mathcal{U}_{\Gamma_{0}}^{h_{n}}-\mathcal{U}_{\Gamma_{0}}^{h_{n}}\right\|_{H^{1}(\Omega)}=0 .
\end{aligned}
$$

Thus, we obtain

$$
\lim _{n \rightarrow \infty} \lim _{m \rightarrow \infty} A_{4}=0
$$

Finally, we also get that

$$
\begin{aligned}
\lim _{n \rightarrow \infty} \lim _{m \rightarrow \infty} A_{5} & :=\lim _{n \rightarrow \infty} \lim _{m \rightarrow \infty}\left(f_{n_{m}}-f_{n}, \mathcal{U}_{\Gamma_{n_{m}}}^{h_{n}}\right) \\
& =\lim _{n \rightarrow \infty} \lim _{m \rightarrow \infty}\left(f_{n_{m}}-f_{n}, \mathcal{U}_{\Gamma_{0}}^{h_{n}}\right)+\lim _{n \rightarrow \infty} \lim _{m \rightarrow \infty}\left(f_{n_{m}}-f_{n}, \mathcal{U}_{\Gamma_{n_{m}}}^{h_{n}}-\mathcal{U}_{\Gamma_{0}}^{h_{n}}\right) \\
& \leq \lim _{n \rightarrow \infty}\left(f_{0}-f_{n}, \mathcal{U}_{\Gamma_{0}}^{h_{n}}\right)+C \lim _{n \rightarrow \infty} \lim _{m \rightarrow \infty}\left\|\mathcal{U}_{\Gamma_{n_{m}}}^{h_{n}}-\mathcal{U}_{\Gamma_{0}}^{h_{n}}\right\|_{H^{1}(\Omega)}=0
\end{aligned}
$$

and

$$
\begin{aligned}
\lim _{n \rightarrow \infty} \lim _{m \rightarrow \infty} A_{6} & :=\lim _{n \rightarrow \infty} \lim _{m \rightarrow \infty}\left\langle g_{n_{m}}-g_{n}, \gamma \mathcal{U}_{\Gamma_{n_{m}}}^{h_{n}}\right\rangle \\
& =\lim _{n \rightarrow \infty} \lim _{m \rightarrow \infty}\left\langle g_{n_{m}}-g_{n}, \gamma\left(\mathcal{U}_{\Gamma_{n_{m}}}^{h_{n}}-\mathcal{U}_{\Gamma_{0}}^{h_{n}}\right)\right\rangle \\
& \leq C \lim _{n \rightarrow \infty} \lim _{m \rightarrow \infty}\left\|\gamma\left(\mathcal{U}_{\Gamma_{n_{m}}}^{h_{n}}-\mathcal{U}_{\Gamma_{0}}^{h_{n}}\right)\right\|_{L^{2}(\partial \Omega)} \\
& \leq C \lim _{n \rightarrow \infty} \lim _{m \rightarrow \infty}\left\|\mathcal{U}_{\Gamma_{n_{m}}}^{h_{n}}-\mathcal{U}_{\Gamma_{0}}^{h_{n}}\right\|_{H^{1}(\Omega)}=0 .
\end{aligned}
$$

Therefore, it follows from the equations $3.8-3.14$ that

$$
\lim _{n \rightarrow \infty} \lim _{m \rightarrow \infty} \mathcal{J}_{\delta_{n}}^{h_{n}{ }^{\prime}}\left(\Gamma_{n_{m}}\right)\left(\Gamma_{n_{m}}-\Gamma_{n}\right)=0 .
$$

Combining this with 3.7, we obtain that $\mathcal{U}_{\Gamma_{0}}=\mathcal{U}_{\Gamma^{\dagger}}$. Then, by the definition of $\Gamma^{\dagger}$, the weakly l.s.c. property of $\mathcal{R}$ and (3.5), we get

$$
\mathcal{R}\left(\Gamma^{\dagger}\right) \leq \mathcal{R}\left(\Gamma_{0}\right) \leq \liminf _{n} \mathcal{R}\left(\Gamma_{n}\right) \leq \limsup _{n} \mathcal{R}\left(\Gamma_{n}\right) \leq \mathcal{R}\left(\Gamma^{\dagger}\right) .
$$

Thus, $\mathcal{R}\left(\Gamma^{\dagger}\right)=\mathcal{R}\left(\Gamma_{0}\right)=\lim _{n \rightarrow \infty} \mathcal{R}\left(\Gamma_{n}\right)$. By the uniqueness of $\Gamma^{\dagger}$, we have $\Gamma_{0}=\Gamma^{\dagger}$. Furthermore, since $\left(\Gamma_{n}\right)_{n}$ weakly converges in $\mathbf{L}_{\text {sym }}^{2}(\Omega) \times L^{2}(\Omega) \times L^{2}(\partial \Omega)$ to $\Gamma_{0}$, we conclude from the last equation that $\left(\Gamma_{n}\right)_{n}$ converges to $\Gamma_{0}$ in the $\mathbf{L}_{\text {sym }}^{2}(\Omega) \times L^{2}(\Omega) \times L^{2}(\partial \Omega)$-norm.

It remains to show that the sequence $\left(\mathcal{U}_{\Gamma_{n}}^{h_{n}}\right)_{n}$ converges to $\Phi^{\dagger}=\mathcal{U}_{\Gamma^{\dagger}}$ in the $H^{1}(\Omega)$-norm. We first get from (2.7) that

$$
\lim _{n \rightarrow \infty}\left\|\mathcal{U}_{\Gamma^{\dagger}}-\mathcal{U}_{\Gamma^{\dagger}}^{h_{n}}\right\|_{H^{1}(\Omega)}=0
$$


Furthermore, in view of 2.12 we also have that

$$
\begin{aligned}
\frac{C_{\Omega} \underline{q}}{1+C_{\Omega}}\left\|\mathcal{U}_{\Gamma_{n}}^{h_{n}}-\mathcal{U}_{\Gamma^{\dagger}}^{h_{n}}\right\|_{H^{1}(\Omega)}^{2} \leq & \int_{\Omega}\left(Q^{\dagger}-Q_{n}\right) \nabla \mathcal{U}_{\Gamma^{\dagger}}^{h_{n}} \cdot \nabla\left(\mathcal{U}_{\Gamma_{n}}^{h_{n}}-\mathcal{U}_{\Gamma^{\dagger}}^{h_{n}}\right) \\
& +\left(f_{n}-f^{\dagger}, \mathcal{U}_{\Gamma_{n}}^{h_{n}}-\mathcal{U}_{\Gamma^{\dagger}}^{h_{n}}\right)+\left\langle g_{n}-g^{\dagger}, \gamma\left(\mathcal{U}_{\Gamma_{n}}^{h_{n}}-\mathcal{U}_{\Gamma^{\dagger}}^{h_{n}}\right)\right\rangle .
\end{aligned}
$$

Since $f_{n} \rightarrow f^{\dagger}$ in the $L^{2}(\Omega)$-norm and $g_{n} \rightarrow g^{\dagger}$ in the $L^{2}(\partial \Omega)$-norm together with the uniform boundedness (2.5), it follows that

$$
\lim _{n \rightarrow \infty}\left(\left(f_{n}-f^{\dagger}, \mathcal{U}_{\Gamma_{n}}^{h_{n}}-\mathcal{U}_{\Gamma^{\dagger}}^{h_{n}}\right)+\left\langle g_{n}-g^{\dagger}, \gamma\left(\mathcal{U}_{\Gamma_{n}}^{h_{n}}-\mathcal{U}_{\Gamma^{\dagger}}^{h_{n}}\right)\right\rangle\right)=0 .
$$

We now rewrite

$$
\begin{aligned}
\int_{\Omega} & \left(Q^{\dagger}-Q_{n}\right) \nabla \mathcal{U}_{\Gamma^{\dagger}}^{h_{n}} \cdot \nabla\left(\mathcal{U}_{\Gamma_{n}}^{h_{n}}-\mathcal{U}_{\Gamma^{\dagger}}^{h_{n}}\right) \\
\quad= & \int_{\Omega}\left(Q^{\dagger}-Q_{n}\right) \nabla \mathcal{U}_{\Gamma^{\dagger}} \cdot \nabla\left(\mathcal{U}_{\Gamma_{n}}^{h_{n}}-\mathcal{U}_{\Gamma^{\dagger}}^{h_{n}}\right)+\int_{\Omega}\left(Q^{\dagger}-Q_{n}\right) \nabla\left(\mathcal{U}_{\Gamma^{\dagger}}^{h_{n}}-\mathcal{U}_{\Gamma^{\dagger}}\right) \cdot \nabla\left(\mathcal{U}_{\Gamma_{n}}^{h_{n}}-\mathcal{U}_{\Gamma^{\dagger}}^{h_{n}}\right) .
\end{aligned}
$$

We will estimate for two terms in the right hand side of the above equation. For simplicity of notation we here set

$$
Q^{\dagger}-Q_{n}:=\left(q_{i j}^{n}\right)_{i, j=\overline{1, d}}, \quad \nabla \mathcal{U}_{\Gamma^{\dagger}} \quad:=\left(U_{1}, \ldots, U_{d}\right) \quad \text { and } \quad \nabla\left(\mathcal{U}_{\Gamma_{n}}^{h_{n}}-\mathcal{U}_{\Gamma^{\dagger}}^{h_{n}}\right):=\quad\left(V_{1}^{n}, \ldots, V_{d}^{n}\right) .
$$

Then, we have

$$
\begin{aligned}
\int_{\Omega} & \left(Q^{\dagger}-Q_{n}\right) \nabla \mathcal{U}_{\Gamma^{\dagger}} \cdot \nabla\left(\mathcal{U}_{\Gamma_{n}}^{h_{n}}-\mathcal{U}_{\Gamma^{\dagger}}^{h_{n}}\right)=\int_{\Omega}\left(\sum_{j=1}^{d} q_{1 j}^{n} U_{j}, \ldots, \sum_{j=1}^{d} q_{d j}^{n} U_{j}\right) \cdot\left(V_{1}^{n}, \ldots, V_{d}^{n}\right) \\
\leq & \left(\int_{\Omega}\left(\sum_{j=1}^{d} q_{1 j}^{n} U_{j}\right)^{2}+\ldots+\left(\sum_{j=1}^{d} q_{d j}^{n} U_{j}\right)^{2}\right)^{1 / 2}\left(\int_{\Omega}\left(V_{1}^{n}\right)^{2}+\ldots+\left(V_{d}^{n}\right)^{2}\right)^{1 / 2} \\
\leq & \left(\int_{\Omega}\left(\sum_{i, j=1}^{d}\left(q_{i j}^{n}\right)^{2}\right)\left(\sum_{i=1}^{d} U_{i}^{2}\right)\right)^{1 / 2}\left(\int_{\Omega}\left(V_{1}^{n}\right)^{2}+\ldots+\left(V_{d}^{n}\right)^{2}\right)^{1 / 2} \\
& =\left(\int_{\Omega}\left\|Q^{\dagger}-Q_{n}\right\|_{\mathcal{S}_{d}}^{2}\left|\nabla \mathcal{U}_{\Gamma^{\dagger}}\right|^{2}\right)^{1 / 2}\left(\int_{\Omega}\left|\nabla\left(\mathcal{U}_{\Gamma_{n}}^{h_{n}}-\mathcal{U}_{\Gamma^{\dagger}}^{h_{n}}\right)\right|^{2}\right)^{1 / 2} \\
\leq & \sqrt{2}\left(\int_{\Omega}\left\|Q^{\dagger}-Q_{n}\right\|_{\mathcal{S}_{d}}^{2}\left|\nabla \mathcal{U}_{\Gamma^{\dagger}}\right|^{2}\right)^{1 / 2}\left(\int_{\Omega}\left|\nabla \mathcal{U}_{\Gamma_{n}}^{h_{n}}\right|^{2}+\int_{\Omega}\left|\nabla \mathcal{U}_{\Gamma^{\dagger}}^{h_{n}}\right|^{2}\right)^{1 / 2} \\
\leq & C\left(\mathcal{R}^{2}\left(\Gamma_{n}\right)+\mathcal{R}^{2}\left(\Gamma^{\dagger}\right)\right)^{1 / 2}\left(\int_{\Omega}\left\|Q^{\dagger}-Q_{n}\right\|_{\mathcal{S}_{d}}^{2}\left|\nabla \mathcal{U}_{\Gamma^{\dagger}}\right|^{2}\right)^{1 / 2}, \text { by } 2.5 \\
\leq & C\left(\int_{\Omega}\left\|Q^{\dagger}-Q_{n}\right\|_{\mathcal{S}_{d}}^{2}\left|\nabla \mathcal{U}_{\Gamma^{\dagger}}\right|^{2}\right)^{1 / 2}, \text { by } 3.5 .
\end{aligned}
$$

Similarly, we get

$$
\int_{\Omega}\left(Q^{\dagger}-Q_{n}\right) \nabla\left(\mathcal{U}_{\Gamma^{\dagger}}^{h_{n}}-\mathcal{U}_{\Gamma^{\dagger}}\right) \cdot \nabla\left(\mathcal{U}_{\Gamma_{n}}^{h_{n}}-\mathcal{U}_{\Gamma^{\dagger}}^{h_{n}}\right) \leq C\left\|\mathcal{U}_{\Gamma^{\dagger}}-\mathcal{U}_{\Gamma^{\dagger}}^{h_{n}}\right\|_{H^{1}(\Omega)},
$$

and arrive at

$$
\int_{\Omega}\left(Q^{\dagger}-Q_{n}\right) \nabla \mathcal{U}_{\Gamma^{\dagger}}^{h_{n}} \cdot \nabla\left(\mathcal{U}_{\Gamma_{n}}^{h_{n}}-\mathcal{U}_{\Gamma^{\dagger}}^{h_{n}}\right) \leq C\left(\int_{\Omega}\left\|Q^{\dagger}-Q_{n}\right\|_{\mathcal{S}_{d}}^{2}\left|\nabla \mathcal{U}_{\Gamma^{\dagger}}\right|^{2}\right)^{1 / 2}+C\left\|\mathcal{U}_{\Gamma^{\dagger}}-\mathcal{U}_{\Gamma^{\dagger}}^{h_{n}}\right\|_{H^{1}(\Omega)}
$$

Since $Q_{n} \rightarrow Q^{\dagger}$ in the $\mathbf{L}_{\text {sym }}^{2}(\Omega)$-norm, up to a subsequence we assume that $\left(Q_{n}\right)_{n}$ converges to $Q^{\dagger}$ a.e. in $\Omega$. Then, by the Lebesgue dominated convergence theorem, we deduce that

$$
\lim _{n \rightarrow \infty} \int_{\Omega}\left\|Q^{\dagger}-Q_{n}\right\|_{\mathcal{S}_{d}}^{2}\left|\nabla \mathcal{U}_{\Gamma^{\dagger}}\right|^{2}=0
$$


Thus, together with (3.15), we have

$$
\lim _{n \rightarrow \infty} \int_{\Omega}\left(Q^{\dagger}-Q_{n}\right) \nabla \mathcal{U}_{\Gamma^{\dagger}}^{h_{n}} \cdot \nabla\left(\mathcal{U}_{\Gamma_{n}}^{h_{n}}-\mathcal{U}_{\Gamma^{\dagger}}^{h_{n}}\right)=0 .
$$

It follows from $3.16-3.18$ that $\lim _{n \rightarrow \infty}\left\|\mathcal{U}_{\Gamma_{n}}^{h_{n}}-\mathcal{U}_{\Gamma^{\dagger}}^{h_{n}}\right\|_{H^{1}(\Omega)}=0$. By serving of 3.15 again, we then conclude that $\lim _{n \rightarrow \infty}\left\|\mathcal{U}_{\Gamma_{n}}^{h_{n}}-\mathcal{U}_{\Gamma^{\dagger}}\right\|_{H^{1}(\Omega)}=0$, which finishes the proof.

\section{Error bounds}

In this section we investigate error bounds of discrete regularized solutions to the identification problem. For any $\Gamma:=(Q, f, g) \in \mathcal{H}_{a d}$ the mapping

$$
\mathcal{U}_{\Gamma}^{\prime}: \quad \mathbf{L}_{\mathrm{sym}}^{\infty}(\Omega) \times L^{2}(\Omega) \times L^{2}(\partial \Omega) \quad \rightarrow \quad H_{\diamond}^{1}(\Omega)
$$

is linear, continuous with the dual

$$
\mathcal{U}_{\Gamma}^{\prime *}: H_{\diamond}^{1}(\Omega)^{*} \rightarrow \mathbf{L}_{\mathrm{sym}}^{\infty}(\Omega)^{*} \times L^{2}(\Omega) \times L^{2}(\partial \Omega) .
$$

Theorem 4.1. Assume that a function $w^{*} \in H_{\diamond}^{1}(\Omega)^{*}$ exists such that

$$
\mathcal{U}_{\Gamma^{\dagger}}^{\prime *} w^{*}=\Gamma^{\dagger} \text {. }
$$

Then

$$
\begin{aligned}
\left\|\mathcal{U}_{\Gamma^{h}}^{h}-\mathcal{U}_{\Gamma^{\dagger}}\right\|_{H^{1}(\Omega)}^{2} & +\rho\left\|\Gamma^{h}-\Gamma^{\dagger}\right\|_{\mathbf{L}_{s y m}^{2}(\Omega) \times L^{2}(\Omega) \times L^{2}(\partial \Omega)}^{2} \\
& =\mathcal{O}\left(h^{-2} \delta^{2}+\left(\chi_{\Phi^{\dagger}}^{h}\right)^{2}+\left(\beta_{\mathcal{U}_{\Gamma^{\dagger}}}^{h}\right)^{2}+\left(\chi_{w}^{h}\right)^{2}+\rho^{2}\right),
\end{aligned}
$$

where $\Gamma^{h}:=\left(Q^{h}, f^{h}, g^{h}\right)$ is the unique solution to $\left(\mathcal{P}_{\delta}^{\rho, h}\right)$ and $w \in H_{\diamond}^{1}(\Omega)$ is the unique weak solution of the Neumann problem

$$
-\nabla \cdot\left(Q^{\dagger} \nabla w\right)=f^{\dagger}+w^{*} \quad \text { in } \Omega \text { and } Q^{\dagger} \nabla w \cdot \vec{n}=g^{\dagger} \text { on } \partial \Omega .
$$

Remark 4.2. Due to Remark 3.2 in case $\mathcal{U}_{\Gamma^{\dagger}}, w \in H^{2}(\Omega)$ we have $0 \leq \chi_{\Phi^{\dagger}}^{h}, \beta_{\mathcal{U}_{\Gamma^{\dagger}}}^{h}, \chi_{w}^{h} \leq C h$. Therefore, with $\delta \sim h^{2}$ and $\rho \sim h$ we obtain the following error bounds

$$
\begin{aligned}
& \left\|\mathcal{U}_{\Gamma^{h}}^{h}-\mathcal{U}_{\Gamma^{\dagger}}\right\|_{H^{1}(\Omega)}=\mathcal{O}(h) \quad \text { and } \\
& \left\|\Gamma^{h}-\Gamma^{\dagger}\right\|_{\mathbf{L}_{\text {sym }}^{2}(\Omega) \times L^{2}(\Omega) \times L^{2}(\partial \Omega)}=\mathcal{O}\left(h^{1 / 2}\right) .
\end{aligned}
$$

Remark 4.3. Let $\bar{\Gamma}:=(\bar{Q}, \bar{f}, \bar{g}) \in \mathcal{I}\left(\Phi^{\dagger}\right)$ be such that the equation 4.1) satisfying with $\bar{\Gamma}$ for some $w^{*} \in H_{\diamond}^{1}(\Omega)^{*}$, i.e. $\mathcal{U}_{\bar{\Gamma}}^{\prime *} w^{*}=\bar{\Gamma}$. Then $\bar{\Gamma}$ is the unique minimum norm solution of the identification, i.e. $\bar{\Gamma}=\Gamma^{\dagger}$.

Indeed, due to 2.2 we have for all $\Gamma:=(Q, f, g) \in \mathcal{I}\left(\Phi^{\dagger}\right)$ that

$$
\begin{aligned}
\mho:=(\bar{\Gamma}, \Gamma-\bar{\Gamma})_{\mathbf{L}_{\mathrm{sym}}^{2}(\Omega) \times L^{2}(\Omega) \times L^{2}(\partial \Omega)} & =\langle\bar{\Gamma}, \Gamma-\bar{\Gamma}\rangle_{\left(\mathbf{L}_{\mathrm{sym}}^{\infty}(\Omega)^{*} \times L^{2}(\Omega) \times L^{2}(\partial \Omega), \mathbf{L}_{\mathrm{sym}}^{\infty}(\Omega) \times L^{2}(\Omega) \times L^{2}(\partial \Omega)\right)} \\
& =\left\langle\mathcal{U}_{\bar{\Gamma}}^{\prime *} w^{*}, \Gamma-\bar{\Gamma}\right\rangle_{\left(\mathbf{L}_{\mathrm{sym}}^{\infty}(\Omega)^{*} \times L^{2}(\Omega) \times L^{2}(\partial \Omega), \mathbf{L}_{\mathrm{sym}}^{\infty}(\Omega) \times L^{2}(\Omega) \times L^{2}(\partial \Omega)\right)} \\
& =\left\langle w^{*}, \mathcal{U}_{\bar{\Gamma}}^{\prime}(\Gamma-\bar{\Gamma})\right\rangle_{\left(H^{1}(\Omega)^{*}, H^{1}(\Omega)\right)} \\
& =\int_{\Omega} \bar{Q} \nabla \mathcal{U}_{\bar{\Gamma}}^{\prime}(\Gamma-\bar{\Gamma}) \cdot \nabla W
\end{aligned}
$$

for some $W \in H_{\diamond}^{1}(\Omega)$, since the expression $[u, v]:=\int_{\Omega} \bar{Q} \nabla u \cdot \nabla v$ generates a scalar inner product on the space $H_{\diamond}^{1}(\Omega)$ which is equivalent to the usual one. By 2.1 we then get

$$
\begin{aligned}
\mho & =-\int_{\Omega}(Q-\bar{Q}) \nabla \mathcal{U}_{\bar{\Gamma}} \cdot \nabla W+(f-\bar{f}, W)+\langle g-\bar{g}, \gamma W\rangle \\
& =\int_{\Omega} \bar{Q} \nabla \mathcal{U}_{\bar{\Gamma}} \cdot \nabla W-(\bar{f}, W)-\langle\bar{g}, \gamma W\rangle-\left(\int_{\Omega} Q \nabla \mathcal{U}_{\bar{\Gamma}} \cdot \nabla W-(f, W)-\langle g, \gamma W\rangle\right)=0,
\end{aligned}
$$


due to 1.6 and the fact $\mathcal{U}_{\bar{\Gamma}}=\mathcal{U}_{\Gamma}=\Phi^{\dagger}$. Therefore, we deduce that

$$
\begin{aligned}
\frac{1}{2}\|\Gamma\|_{\mathbf{L}_{\text {sym }}^{2}(\Omega) \times L^{2}(\Omega) \times L^{2}(\partial \Omega)}^{2} & -\frac{1}{2}\|\bar{\Gamma}\|_{\mathbf{L}_{\text {sym }}^{2}(\Omega) \times L^{2}(\Omega) \times L^{2}(\partial \Omega)}^{2} \\
& =\frac{1}{2}\|\Gamma-\bar{\Gamma}\|_{\mathbf{L}_{\text {sym }}^{2}(\Omega) \times L^{2}(\Omega) \times L^{2}(\partial \Omega)}^{2}+\mho \geq 0,
\end{aligned}
$$

which completed the proof.

Proof of Theorem 4.1. Due to the optimality of $\Gamma^{h}$, we get that

$$
\mathcal{J}_{\delta}^{h}\left(\Gamma^{h}\right)+\rho \mathcal{R}\left(\Gamma^{h}\right) \leq \mathcal{J}_{\delta}^{h}\left(\Gamma^{\dagger}\right)+\rho \mathcal{R}\left(\Gamma^{\dagger}\right)
$$

which implies

$$
\begin{aligned}
\mathcal{J}_{\delta}^{h} & \left(\Gamma^{h}\right)+\rho\left\|\Gamma^{h}-\Gamma^{\dagger}\right\|_{\mathbf{L}_{\text {sym }}^{2}(\Omega) \times L^{2}(\Omega) \times L^{2}(\partial \Omega)}^{2} \\
& \leq \mathcal{J}_{\delta}^{h}\left(\Gamma^{\dagger}\right)+2 \rho\left\langle\Gamma^{\dagger}, \Gamma^{\dagger}-\Gamma^{h}\right\rangle_{\mathbf{L}_{\text {sym }}^{2}(\Omega) \times L^{2}(\Omega) \times L^{2}(\partial \Omega)} \\
& \leq C\left(h^{-2} \delta^{2}+\left(\chi_{\Phi^{\dagger}}^{h}\right)^{2}+\left(\beta_{\mathcal{U}_{\Gamma^{\dagger}}}\right)^{2}\right)+2 \rho\left(\Gamma^{\dagger}, \Gamma^{\dagger}-\Gamma^{h}\right)_{\mathbf{L}_{\text {sym }}^{2}(\Omega) \times L^{2}(\Omega) \times L^{2}(\partial \Omega)},
\end{aligned}
$$

by Lemma 3.3 . Now, by $(2.2)$ and 4.1 , we infer that

$$
I:=\left(\Gamma^{\dagger}, \Gamma^{\dagger}-\Gamma^{h}\right)_{\mathbf{L}_{\mathrm{sym}}^{2}(\Omega) \times L^{2}(\Omega) \times L^{2}(\partial \Omega)}=\left\langle w^{*}, \mathcal{U}_{\Gamma^{\dagger}}^{\prime}\left(\Gamma^{\dagger}-\Gamma^{h}\right)\right\rangle_{\left(H^{1}(\Omega)^{*}, H^{1}(\Omega)\right)} .
$$

Thus, by the definition of the weak solution to 4.3 and $(2.1)$, we obtain

$$
\begin{aligned}
& I=\int_{\Omega} Q^{\dagger} \nabla \mathcal{U}_{\Gamma^{\dagger}}^{\prime}\left(\Gamma^{\dagger}-\Gamma^{h}\right) \cdot \nabla w \underbrace{-\left(f^{\dagger}-\Gamma^{h}\right), \text { by } \sqrt{1.6},}_{-\int_{\Omega} Q^{\dagger} \nabla \mathcal{U}_{\Gamma^{\dagger}} \cdot \nabla \mathcal{U}_{\Gamma^{\dagger}}^{\prime}} \\
& =\int_{\Omega} Q^{\dagger} \nabla \mathcal{U}_{\Gamma^{\dagger}}^{\prime}\left(\Gamma^{\dagger}-\Gamma^{h}\right) \cdot \nabla\left(w-\mathcal{U}_{\Gamma^{\dagger}}\right) \\
& =-\int_{\Omega}\left(Q^{\dagger}-Q^{h}\right) \nabla \mathcal{U}_{\Gamma^{\dagger}} \cdot \nabla\left(w-\mathcal{U}_{\Gamma^{\dagger}}\right)+\left(f^{\dagger}-f^{h}, w-\mathcal{U}_{\Gamma^{\dagger}}\right)+\left\langle g^{\dagger}-g^{h}, \gamma\left(w-\mathcal{U}_{\Gamma^{\dagger}}\right)\right\rangle \\
& =\underbrace{-\int_{\Omega} Q^{\dagger} \nabla \mathcal{U}_{\Gamma^{\dagger}} \cdot \nabla\left(w-\mathcal{U}_{\Gamma^{\dagger}}\right)+\left(f^{\dagger}, w-\mathcal{U}_{\Gamma^{\dagger}}\right)+\left\langle g^{\dagger}, \gamma\left(w-\mathcal{U}_{\Gamma^{\dagger}}\right)\right\rangle}_{=0, \text { by } 1.6,} \\
& +\int_{\Omega} Q^{h} \nabla \mathcal{U}_{\Gamma^{\dagger}} \cdot \nabla\left(w-\mathcal{U}_{\Gamma^{\dagger}}\right) \underbrace{-\left(f^{h}, w-\mathcal{U}_{\Gamma^{\dagger}}\right)-\left\langle g^{h}, \gamma\left(w-\mathcal{U}_{\Gamma^{\dagger}}\right)\right\rangle}_{-\int_{\Omega} Q^{h} \nabla \mathcal{U}_{\Gamma^{h}} \cdot \nabla\left(w-\mathcal{U}_{\Gamma^{\dagger}}\right)} \\
& =\int_{\Omega} Q^{h} \nabla\left(\mathcal{U}_{\Gamma^{\dagger}}-\mathcal{U}_{\Gamma^{h}}\right) \cdot \nabla\left(w-\mathcal{U}_{\Gamma^{\dagger}}\right)
\end{aligned}
$$

which yields

$$
\begin{aligned}
I= & \int_{\Omega} Q^{h} \nabla\left(\mathcal{U}_{\Gamma^{\dagger}}-\Pi^{h} z_{\delta}\right) \cdot \nabla\left(w-\mathcal{U}_{\Gamma^{\dagger}}\right)+\int_{\Omega} Q^{h} \nabla\left(\mathcal{U}_{\Gamma^{h}}^{h}-\mathcal{U}_{\Gamma^{h}}\right) \cdot \nabla\left(w-\mathcal{U}_{\Gamma^{\dagger}}\right) \\
& +\int_{\Omega} Q^{h} \nabla\left(\Pi^{h} z_{\delta}-\mathcal{U}_{\Gamma^{h}}^{h}\right) \cdot \nabla\left(w-\mathcal{U}_{\Gamma^{\dagger}}\right):=\quad I_{1}+I_{2}+I_{3} .
\end{aligned}
$$

For $I_{1}$ we have from 3.2 that

$$
I_{1}:=\int_{\Omega} Q^{h} \nabla\left(\mathcal{U}_{\Gamma^{\dagger}}-\Pi^{h} z_{\delta}\right) \cdot \nabla\left(w-\mathcal{U}_{\Gamma^{\dagger}}\right) \leq C\left\|\mathcal{U}_{\Gamma^{\dagger}}-\Pi^{h} z_{\delta}\right\|_{H^{1}(\Omega)} \leq C h^{-1} \delta+\chi_{\Phi^{\dagger}}^{h} .
$$

Due to 1.6 and 2.4 , we get $\int_{\Omega} Q^{h} \nabla\left(\mathcal{U}_{\Gamma^{h}}^{h}-\mathcal{U}_{\Gamma^{h}}\right) \cdot \nabla \Pi^{h}\left(w-\mathcal{U}_{\Gamma^{\dagger}}\right)=0$ and then infer that

$$
\begin{aligned}
I_{2} & :=\int_{\Omega} Q^{h} \nabla\left(\mathcal{U}_{\Gamma^{h}}^{h}-\mathcal{U}_{\Gamma^{h}}\right) \cdot \nabla\left(w-\mathcal{U}_{\Gamma^{\dagger}}\right) \\
& =\int_{\Omega} Q^{h} \nabla\left(\mathcal{U}_{\Gamma^{h}}^{h}-\mathcal{U}_{\Gamma^{h}}\right) \cdot \nabla\left(w-\mathcal{U}_{\Gamma^{\dagger}}-\Pi^{h}\left(w-\mathcal{U}_{\Gamma^{\dagger}}\right)\right) \\
& \leq C\left(\left\|w-\Pi^{h} w\right\|_{H^{1}(\Omega)}+\left\|\mathcal{U}_{\Gamma^{\dagger}}-\Pi^{h} \mathcal{U}_{\Gamma^{\dagger}}\right\|_{H^{1}(\Omega)}\right) \leq C\left(\chi_{w}^{h}+\chi_{\Phi^{\dagger}}^{h}\right) .
\end{aligned}
$$


Finally, we have that

$$
\begin{aligned}
I_{3} & :=\int_{\Omega} Q^{h} \nabla\left(\Pi^{h} z_{\delta}-\mathcal{U}_{\Gamma^{h}}^{h}\right) \cdot \nabla\left(w-\mathcal{U}_{\Gamma^{\dagger}}\right) \\
& \leq\left(\int_{\Omega} Q^{h} \nabla\left(\mathcal{U}_{\Gamma^{h}}^{h}-\Pi^{h} z_{\delta}\right) \cdot \nabla\left(\mathcal{U}_{\Gamma^{h}}^{h}-\Pi^{h} z_{\delta}\right)\right)^{1 / 2} \cdot\left(\int_{\Omega} Q^{h} \nabla\left(w-\mathcal{U}_{\Gamma^{\dagger}}\right) \cdot \nabla\left(w-\mathcal{U}_{\Gamma^{\dagger}}\right)\right)^{1 / 2} \\
& \leq \underbrace{\frac{1}{4 \rho} \int_{\Omega} Q^{h} \nabla\left(\mathcal{U}_{\Gamma^{h}}^{h}-\Pi^{h} z_{\delta}\right) \cdot \nabla\left(\mathcal{U}_{\Gamma^{h}}^{h}-\Pi^{h} z_{\delta}\right)}_{\mathcal{J}_{\delta}^{h}\left(\Gamma^{h}\right)}+\rho \int_{\Omega} Q^{h} \nabla\left(w-\mathcal{U}_{\Gamma^{\dagger}}\right) \cdot \nabla\left(w-\mathcal{U}_{\Gamma^{\dagger}}\right) \\
& \leq \frac{1}{4 \rho} \mathcal{J}_{\delta}^{h}\left(\Gamma^{h}\right)+C \rho .
\end{aligned}
$$

It follows from (4.8)-4.11) that

$$
I \leq C\left(h^{-1} \delta+\chi_{\Phi^{\dagger}}^{h}+\chi_{w}^{h}+\rho\right)+\frac{1}{4 \rho} \mathcal{J}_{\delta}^{h}\left(\Gamma^{h}\right) .
$$

Thus, together with 4.6 - 4.7), we get

$$
\frac{1}{2} \mathcal{J}_{\delta}^{h}\left(\Gamma^{h}\right)+\rho\left\|\Gamma^{h}-\Gamma^{\dagger}\right\|_{\mathbf{L}_{\mathrm{sym}}^{2}(\Omega) \times L^{2}(\Omega) \times L^{2}(\partial \Omega)}^{2} \leq C\left(h^{-2} \delta^{2}+\left(\chi_{\Phi^{\dagger}}^{h}\right)^{2}+\left(\beta_{\mathcal{U}_{\Gamma^{\dagger}}}^{h}\right)^{2}+\left(\chi_{w}^{h}\right)^{2}+\rho^{2}\right),
$$

which finishes the proof.

\section{Gradient projection algorithm with Armijo steplength rule}

In this section we present the gradient projection algorithm with Armijo steplength rule (cf. 31, 42]) for numerical solution of the minimization problem $\left(\mathcal{P}_{\delta}^{\rho, h}\right)$.

We first note that for each $\Gamma:=(Q, f, g) \in \mathcal{H}_{a d}$, in view of $(2.14)$, the $\mathcal{L}^{2}$-gradient of the strictly convex cost function $\Upsilon_{\delta}^{\rho, h}$ of the problem $\left(\mathcal{P}_{\delta}^{\rho, h}\right)$ is given by $\nabla \Upsilon_{\delta}^{\rho, h}(\Gamma):=\left(\Upsilon_{Q}(\Gamma), \Upsilon_{f}(\Gamma), \Upsilon_{g}(\Gamma)\right)$ with

$$
\begin{aligned}
& \Upsilon_{Q}(\Gamma)=\nabla \bar{\Pi}^{h} z_{\delta} \otimes \nabla \bar{\Pi}^{h} z_{\delta}-\nabla \mathcal{U}_{\Gamma}^{h} \otimes \nabla \mathcal{U}_{\Gamma}^{h}+2 \rho Q, \\
& \Upsilon_{f}(\Gamma)=2\left(\mathcal{U}_{\Gamma}^{h}-\bar{\Pi}^{h} z_{\delta}+\rho f\right), \\
& \Upsilon_{g}(\Gamma)=2\left(\gamma\left(\mathcal{U}_{\Gamma}^{h}-\bar{\Pi}^{h} z_{\delta}\right)+\rho g\right)
\end{aligned}
$$

and $\bar{\Pi}^{h}$ generating from $\Pi^{h}$ according to 2.13 .

The algorithm is then read as: given a step size control $\beta \in(0,1)$, an initial approximation (cf. Remark 2.6) $\Gamma_{0}:=\left(Q_{0}, f_{0}, g_{0}\right) \in \mathcal{H}_{a d} \cap\left(\mathcal{V}_{0}^{h}{ }^{d \times d} \times \mathcal{V}_{1}^{h} \times \mathcal{E}_{1}^{h}\right)$, number of iteration $N$ and setting $k=0$.

1. Compute $\mathcal{U}_{\Gamma_{k}}^{h}$ from the variational equation

$$
\int_{\Omega} Q_{k} \nabla \mathcal{U}_{\Gamma_{k}}^{h} \cdot \nabla \varphi^{h}=\left(f_{k}, \varphi^{h}\right)+\left\langle g_{k}, \gamma \varphi^{h}\right\rangle \text { for all } \varphi^{h} \in \mathcal{V}_{1}^{h}
$$

as well as

$$
\begin{aligned}
\Upsilon_{\rho, \delta}^{h}\left(\Gamma_{k}\right)= & \int_{\Omega} Q_{k} \nabla\left(\mathcal{U}_{\Gamma_{k}}^{h}-\bar{\Pi}^{h} z_{\delta}\right) \cdot \nabla\left(\mathcal{U}_{\Gamma_{k}}^{h}-\bar{\Pi}^{h} z_{\delta}\right) \\
& +\rho\left(\left\|Q_{k}\right\|_{\mathbf{L}_{\text {sym }}^{2}(\Omega)}^{2}+\left\|f_{k}\right\|_{L^{2}(\Omega)}^{2}+\left\|g_{k}\right\|_{L^{2}(\partial \Omega)}^{2}\right) .
\end{aligned}
$$

2. Compute the gradient $\nabla \Upsilon_{\delta}^{\rho, h}\left(\Gamma_{k}\right):=\left(\Upsilon_{Q_{k}}\left(\Gamma_{k}\right), \Upsilon_{f_{k}}\left(\Gamma_{k}\right), \Upsilon_{g_{k}}\left(\Gamma_{k}\right)\right)$ with

$$
\begin{aligned}
& \Upsilon_{Q_{k}}\left(\Gamma_{k}\right)=\nabla \bar{\Pi}^{h} z_{\delta} \otimes \nabla \bar{\Pi}^{h} z_{\delta}-\nabla \mathcal{U}_{\Gamma_{k}}^{h} \otimes \nabla \mathcal{U}_{\Gamma_{k}}^{h}+2 \rho Q_{k}, \\
& \Upsilon_{f_{k}}\left(\Gamma_{k}\right)=2\left(\mathcal{U}_{\Gamma_{k}}^{h}-\bar{\Pi}^{h} z_{\delta}+\rho f_{k}\right), \\
& \Upsilon_{g_{k}}\left(\Gamma_{k}\right)=2\left(\gamma\left(\mathcal{U}_{\Gamma_{k}}^{h}-\bar{\Pi}^{h} z_{\delta}\right)+\rho g_{k}\right) .
\end{aligned}
$$


3. Set $\widetilde{\Gamma}_{k}:=\left(\widetilde{Q}_{k}, \widetilde{f}_{k}, \widetilde{g}_{k}\right)$ with $\widetilde{Q}_{k}(x):=P_{\mathcal{K}}\left(Q_{k}(x)-\beta \Upsilon_{Q_{k}}\left(\Gamma_{k}\right)(x)\right), \widetilde{f}_{k}(x):=f_{k}(x)-\beta \Upsilon_{f_{k}}\left(\Gamma_{k}\right)(x)$ and $\widetilde{g}_{k}(x):=g_{k}(x)-\beta \Upsilon_{g_{k}}\left(\Gamma_{k}\right)(x)$.

(a) Compute $\mathcal{U}_{\widetilde{\Gamma}_{k}}^{h}$ according to $5.1, \Upsilon_{\rho, \delta}^{h}\left(\widetilde{\Gamma}_{k}\right)$ according to 5.2 , and with $\tau=10^{-4}$

$$
L:=\Upsilon_{\rho, \delta}^{h}\left(\widetilde{\Gamma}_{k}\right)-\Upsilon_{\rho, \delta}^{h}\left(\Gamma_{k}\right)+\tau \beta\left(\left\|\widetilde{Q}_{k}-Q_{k}\right\|_{\mathbf{L}_{\text {sym }}^{2}(\Omega)}^{2}+\left\|\widetilde{f}_{k}-f_{k}\right\|_{L^{2}(\Omega)}^{2}+\left\|\widetilde{g}_{k}-g_{k}\right\|_{L^{2}(\partial \Omega)}^{2}\right) .
$$

(b) If $L \leq 0$

go to the next step (c) below else set $\beta:=\frac{\beta}{2}$ and then go back (a)

(c) Update $\Gamma_{k}=\widetilde{\Gamma}_{k}$, set $k=k+1$.

4. Compute

$$
\text { Tolerance }:=\left\|\nabla \Upsilon_{\rho, \delta}^{h}\left(\Gamma_{k}\right)\right\|_{\mathbf{L}_{\text {sym }}^{2}(\Omega) \times L^{2}(\Omega) \times L^{2}(\partial \Omega)}-\tau_{1}-\tau_{2}\left\|\nabla \Upsilon_{\rho, \delta}^{h}\left(\Gamma_{0}\right)\right\|_{\mathbf{L}_{\text {sym }}^{2}(\Omega) \times L^{2}(\Omega) \times L^{2}(\partial \Omega)}
$$

with $\tau_{1}:=10^{-3} h$ and $\tau_{2}:=10^{-2} h$. If Tolerance $\leq 0$ or $k>N$, then stop; otherwise go back Step 1 .

\section{Numerical implementation}

For illustrating the theoretical result we consider the Neumann problem

$$
\begin{aligned}
-\nabla \cdot\left(Q^{\dagger} \nabla \Phi^{\dagger}\right) & =f^{\dagger} \text { in } \Omega, \\
Q^{\dagger} \nabla \Phi^{\dagger} \cdot \vec{n} & =g^{\dagger} \text { on } \partial \Omega
\end{aligned}
$$

with $\Omega=\left\{x=\left(x_{1}, x_{2}\right) \in R^{2} \mid-1<x_{1}, x_{2}<1\right\}$.

The special constants in the equation $\sqrt{1.3}$ are chosen as $\underline{q}=0.05$ and $\bar{q}=10$. For discretization we divide the interval $(-1,1)$ into $\ell$ equal segments, and so the domain $\Omega=(-1,1)^{2}$ is divided into $2 \ell^{2}$ triangles, where the diameter of each triangle is $h_{\ell}=\frac{\sqrt{8}}{\ell}$.

We assume that entries of the symmetric diffusion matrix $Q^{\dagger}$ are discontinuous which are defined as

$$
q_{11}^{\dagger}:=2 \chi_{\Omega_{11}}+\chi_{\Omega \backslash \Omega_{11}}, \quad q_{12}^{\dagger}=q_{21}^{\dagger}:=\chi_{\Omega_{12}} \text { and } q_{22}^{\dagger}:=3 \chi_{\Omega_{22}}+2 \chi_{\Omega \backslash \Omega_{22}},
$$

where $\chi_{D}$ is the characteristic functional of the Lebesgue measurable set $D$ and

$$
\begin{aligned}
& \Omega_{11}:=\left\{\left(x_{1}, x_{2}\right) \in \Omega|| x_{1} \mid \leq 3 / 4 \text { and }\left|x_{2}\right| \leq 3 / 4\right\}, \\
& \Omega_{12}:=\left\{\left(x_{1}, x_{2}\right) \in \Omega|| x_{1}|+| x_{2} \mid \leq 3 / 4\right\} \text { and } \\
& \Omega_{22}:=\left\{\left(x_{1}, x_{2}\right) \in \Omega \mid x_{1}^{2}+x_{2}^{2} \leq 9 / 16\right\} .
\end{aligned}
$$

The source functional $f^{\dagger}$ is assumed to be also discontinuous and defined as

$$
f^{\dagger}:=\frac{93-2 \pi}{48} \chi_{\Omega_{1}}+\frac{45-2 \pi}{48} \chi_{\Omega_{2}}-\frac{3+2 \pi}{48} \chi_{\Omega \backslash\left(\Omega_{1} \cup \Omega_{2}\right)},
$$

where

$$
\begin{aligned}
& \Omega_{1}:=\left\{\left(x_{1}, x_{2}\right) \in \Omega \mid 9\left(x_{1}+1 / 2\right)^{2}+16\left(x_{2}-1 / 2\right)^{2} \leq 1\right\} \text { and } \\
& \Omega_{2}:=\left\{\left(x_{1}, x_{2}\right) \in \Omega|| x_{1}-1 / 2 \mid \leq 1 / 4 \text { and }\left|x_{2}+1 / 2\right| \leq 1 / 4\right\} .
\end{aligned}
$$

The Neumann boundary condition $g^{\dagger}$ is chosen with

$$
\begin{aligned}
g^{\dagger}:= & -2 \chi_{[-1,0] \times\{-1\}}+\chi_{(0,1] \times\{-1\}}-\chi_{[-1,0] \times\{1\}}+2 \chi_{(0,1] \times\{1\}} \\
& +3 \chi_{\{-1\} \times(-1,0]}-4 \chi_{\{-1\} \times(0,1)}+4 \chi_{\{1\} \times(-1,0]}-3 \chi_{\{1\} \times(0,1)} .
\end{aligned}
$$


The exact state $\Phi^{\dagger}$ is then computed from the finite element equation $K U=F$, where $K$ and $F$ are the stiffness matrix and the load vector associated with the problem 6.1 - 6.2 , respectively.

We mention that in the above example the sought functions are chosen to be discontinuous. To reconstruct such discontinuous functions one usually employs the total variation regularization which was originally introduced in image denoising by authors of [41. This regularization method was proved to be very effective and analyzed by many authors over the last decades for several ill-posed and inverse problems. We also note that the space of all functions with bounded total variation is a non-reflexive Banach space and the Tikhonovfunction of the total variation regularization is non-differentiable, which cause some certain difficulties in numerically treating for non-linear, ill-posed inverse problems. In the present work the cost function is convex and differentiable, the convergence history given in Table 1 and Table 2 below shows that the algorithm presented in Section 5 performs well for the identification problem with the discontinuous coefficents.

We start the computation with the coarsest level $\ell=3$. To this end, for constructing observations with noise of the exact state $\Phi^{\dagger}$ on this coarsest grid we use

$$
z_{\delta_{\ell}}:=\Phi^{\dagger}+\mathcal{N}_{\overline{\delta_{\ell}}} \text { and } \quad \delta_{\ell}:=\left\|z_{\delta_{\ell}}-\Phi^{\dagger}\right\|_{L^{2}(\Omega)},
$$

where $\overline{\delta_{\ell}}=10 \rho_{\ell}^{1 / 2} h_{\ell}^{3 / 2}, \rho_{\ell}=10^{-3} h_{\ell}$ and $\mathcal{N}_{\overline{\delta_{\ell}}}$ is a $M^{h_{\ell}} \times 1$-matrix of random numbers in the interval $\left(-\overline{\delta_{\ell}}, \overline{\delta_{\ell}}\right), M^{h_{\ell}}=(\ell+1)^{2}$ is the number of nodes of the triangulation $\mathcal{T}^{h_{\ell}}$. Therefore, the exact state $\Phi^{\dagger}$ is only measured at 16 nodes of $\mathcal{T}^{h_{\ell}}$.

We use the algorithm described in $\$ 5$ for computing the numerical solution of the problem $\left(\mathcal{P}_{\rho_{\ell}, \delta_{\ell}}^{h_{\ell}}\right)$. The step size control is chosen with $\beta=0.75$. As the initial approximation we choose

$$
\begin{aligned}
Q_{0} & :=\left[\begin{array}{cc}
2 & 0 \\
0 & 2
\end{array}\right], \quad f_{0}:=\chi_{[-1,0) \times[-1,1]}-\chi_{[0,1] \times[-1,1]} \quad \text { and } \\
g_{0} & :=\chi_{[-1,1] \times\{1\}}-\chi_{[-1,1] \times\{-1\}}+\chi_{\{1\} \times(-1,1)}-\chi_{\{-1\} \times(-1,1)} .
\end{aligned}
$$

At each iteration $k$ we compute Tolerance defined by $(5.3)$. Then the iteration was stopped if Tolerance $\leq 0$ or the number of iterations reached the maximum iteration count of 800 .

After obtaining the numerical solution $\Gamma_{\ell}=\left(Q_{\ell}, f_{\ell}, g_{\ell}\right)$ and the computed numerical state $\mathcal{U}_{\ell}=\mathcal{U}_{\Gamma_{\ell}}^{h_{\ell}}$ of the first iteration process with respect to the coarsest level $\ell=3$, we use their interpolations on the next finer mesh $\ell=6$ as an initial approximation and an observation of the exact state for the algorithm on this finer mesh, i.e. for the next iteration process with respect to the level $\ell=6$ we employ

$$
\left(Q_{0}, f_{0}, g_{0}\right):=I_{1}^{h_{6}} \Gamma_{3} \quad \text { and } \quad z_{\delta_{6}}:=I_{1}^{h_{6}} \mathcal{U}_{3} \quad \text { with } \quad \delta_{6}:=\left\|z_{\delta_{6}}-\Phi^{\dagger}\right\|_{L^{2}(\Omega)}
$$

and $I_{1}^{h_{\ell}}$ being the usual node value interpolation operator on $\mathcal{T}^{h_{\ell}}$, and so on $\ell=12,24, \ldots$ We note that the computation process only requires the measurement data of the exact data for the coarsest level $\ell=3$.

The numerical results are summarized in Table 1 and Table 2, where we present the refinement level $\ell$, mesh size $h_{\ell}$ of the triangulation, regularization parameter $\rho_{\ell}$, noise $\delta_{\ell}$ and number of iterates as well as the final $L^{2}$-error in the coefficients, the final $L^{2}$ and $H^{1}$-error in the states, and their experimental order of convergence (EOC), where $\mathrm{EOC}_{\Phi}:=\frac{\ln \Phi\left(h_{1}\right)-\ln \Phi\left(h_{2}\right)}{\ln h_{1}-\ln h_{2}}$ and $\Phi(h)$ is an error function with respect to $h$.

All figures are here presented corresponding to $\ell=96$. Figure 1 from left to right shows the graphs of $\Phi^{\dagger}$, computed numerical state $\mathcal{U}_{\ell}$ of the algorithm at the last iteration, and the difference to $\Phi^{\dagger}$. In Figure 2 we display the computed numerical source term and boundary condition $f_{\ell}, g_{\ell}$ at the last iteration as well as the differences $f_{\ell}-f^{\dagger}, g_{\ell}-g^{\dagger}$. We write the computed numerical diffusion matrix at the last iteration as

$$
Q_{\ell}:=\left[\begin{array}{ll}
q_{\ell, 11} & q_{\ell, 12} \\
q_{\ell, 12} & q_{\ell, 22}
\end{array}\right] .
$$

Figure 3 then shows $q_{\ell, 11}, q_{\ell, 12}$ and $q_{\ell, 22}$ while Figure 4 shows differences $q_{\ell, 11}-q_{11}^{\dagger}, q_{\ell, 12}-q_{12}^{\dagger}$ and $q_{\ell, 22}-q_{22}^{\dagger}$. For abbreviation we denote by $\Gamma^{\dagger}:=\left(Q^{\dagger}, f^{\dagger}, g^{\dagger}\right)$ and errors

$$
\Delta:=\left\|\Gamma_{\ell}-\Gamma^{\dagger}\right\|_{\mathbf{L}_{\mathrm{sym}}^{2}(\Omega) \times L^{2}(\Omega) \times L^{2}(\partial \Omega)}, \quad \Sigma \quad:=\left\|\mathcal{U}_{\ell}-\Phi^{\dagger}\right\|_{L^{2}(\Omega)} \quad \text { and } \quad \Lambda \quad:=\left\|\mathcal{U}_{\ell}-\Phi^{\dagger}\right\|_{H^{1}(\Omega)} .
$$


Table 1: Refinement level $\ell$, mesh size $h_{\ell}$ of the triangulation, regularization parameter $\rho_{\ell}$, noise $\delta_{\ell}$ and number of iterates.

\begin{tabular}{cllll}
\hline \hline$\ell$ & $h_{\ell}$ & $\rho_{\ell}$ & $\delta_{\ell}$ & Iterate \\
\hline 3 & 0.9428 & $9.4281 \mathrm{e}-4$ & 0.1755 & 800 \\
\hline 6 & 0.4714 & $4.7140 \mathrm{e}-4$ & 0.3847 & 800 \\
\hline 12 & 0.2357 & $2.3570 \mathrm{e}-4$ & 0.3334 & 800 \\
\hline 24 & 0.1179 & $1.1790 \mathrm{e}-4$ & 0.1508 & 800 \\
\hline 48 & $5.8926 \mathrm{e}-2$ & $5.8926 \mathrm{e}-5$ & $6.5163 \mathrm{e}-2$ & 800 \\
\hline 96 & $2.9463 \mathrm{e}-2$ & $2.9463 \mathrm{e}-5$ & $2.9896 \mathrm{e}-2$ & 800 \\
\hline \hline
\end{tabular}

Table 2: Errors $\Delta, \Sigma$ and $\Lambda$ and Experimental order of convergence between finest and coarsest level.

\begin{tabular}{llllll}
\hline \hline$\Delta$ & $\Sigma$ & $\Lambda$ & $\mathbf{E O C}_{\Delta}$ & $\mathbf{E O C}_{\Sigma}$ & $\mathbf{E O C}_{\Lambda}$ \\
\hline 0.6349 & $6.2551 \mathrm{e}-2$ & 0.2789 & - & - & - \\
\hline 0.1974 & $3.7602 \mathrm{e}-2$ & 0.1847 & 1.6854 & 0.7342 & 0.5946 \\
\hline $8.3571 \mathrm{e}-2$ & $1.7066 \mathrm{e}-2$ & 0.1382 & 1.2400 & 1.1397 & 0.4184 \\
\hline $3.1600 \mathrm{e}-2$ & $5.4913 \mathrm{e}-3$ & $6.1769 \mathrm{e}-2$ & 1.4031 & 1.6359 & 1.1618 \\
\hline $1.1524 \mathrm{e}-2$ & $9.4491 \mathrm{e}-4$ & $2.0742 \mathrm{e}-2$ & 1.4553 & 2.5389 & 1.5743 \\
\hline $4.1183 \mathrm{e}-3$ & $2.2575 \mathrm{e}-4$ & $8.9372 \mathrm{e}-3$ & 1.4845 & 2.0655 & 1.2147 \\
\hline Mean & of & EOC & 1.4537 & 1.6228 & 0.9928 \\
\hline \hline
\end{tabular}

Figure 1: Graphs of $\Phi^{\dagger}$, computed numerical state $\mathcal{U}_{\ell}$ of the algorithm at the $800^{\text {th }}$ iteration, and the difference to $\Phi^{\dagger}$.
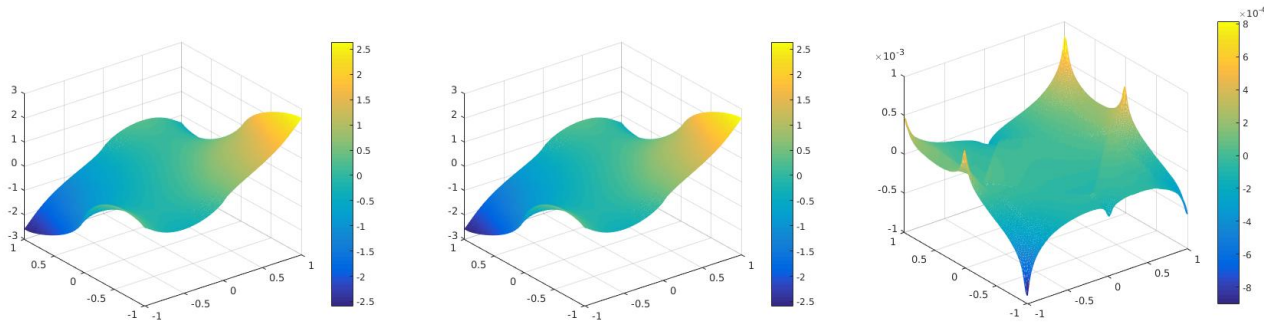

Figure 2: Graphs of $f_{\ell}, g_{\ell}$ at the $800^{\text {th }}$ iteration and the differences $f_{\ell}-f^{\dagger}, g_{\ell}-g^{\dagger}$.

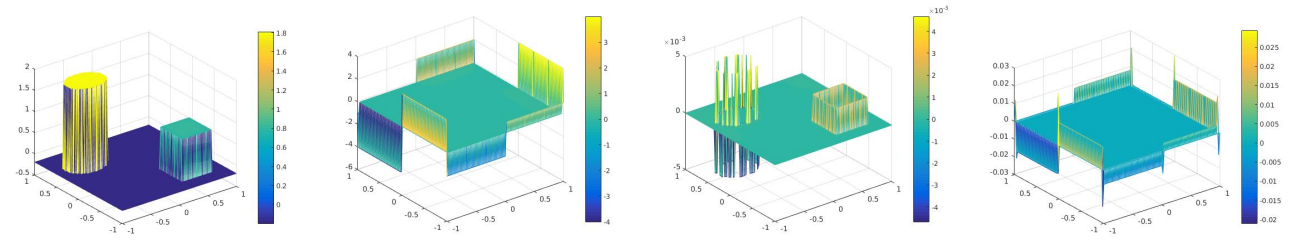


Figure 3: Graphs of $q_{\ell, 11}, q_{\ell, 12}$ and $q_{\ell, 22}$ at the $800^{\text {th }}$ iteration.
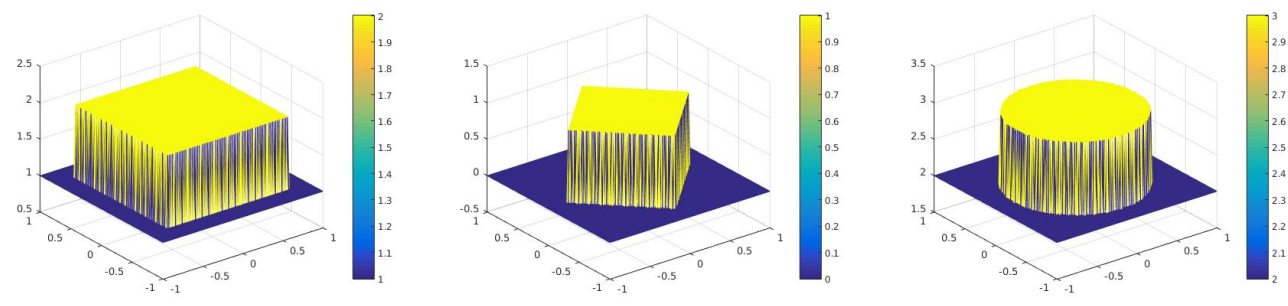

Figure 4: Differences $q_{\ell, 11}-q_{11}^{\dagger}, q_{\ell, 12}-q_{12}^{\dagger}$ and $q_{\ell, 22}-q_{22}^{\dagger}$.
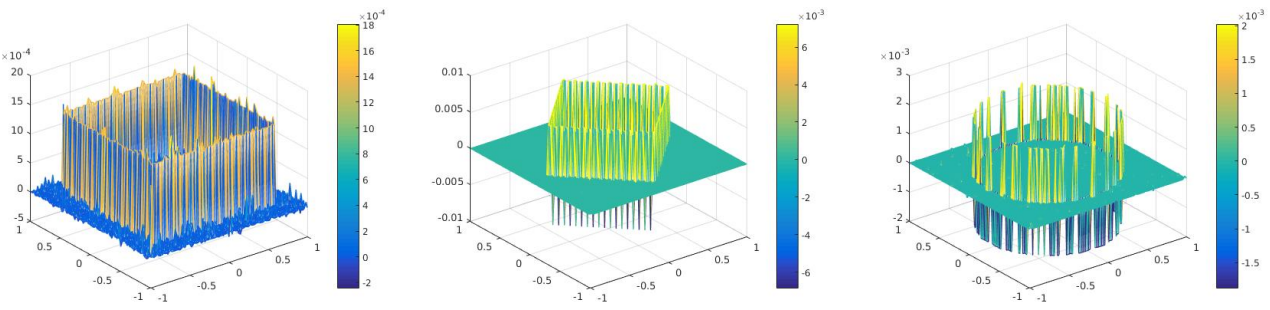

\section{Acknowledgments}

The author would like to thank the Referees and the Editor for their valuable comments and suggestions which helped to improve the present paper.

\section{References}

[1] Attouch H., Buttazzo G. and Michaille G., Variational Analysis in Sobolev and BV Space, Philadelphia: SIAM, 2006.

[2] Baumeister J. and Kunisch K., Identifiability and stability of a two-parameter estimation problem, Appl. Anal. 40, 263-279, 1991.

[3] Banks H. T. and Kunisch K., Estimation Techniques for Distributed Parameter Systems, Systems and Control: Foundations and Applications, Boston: Birkhäuser, 1989.

[4] Bernardi C., Optimal finite element interpolation on curved domain, SIAM J. Numer. Anal. 26, 12121240, 1989.

[5] Bernardi C. and Girault V., A local regularization operator for triangular and quadrilateral finite elements, SIAM J. Numer. Anal. 35, 1893-1916, 1998.

[6] Brenner S. and Scott R., The Mathematical Theory of Finite Element Methods, New York: Springer, 2008.

[7] Chan T. F and Tai X. C., Identification of discontinuous coefficients in elliptic problems using total variation regularization, SIAM J. Sci. Comput. 25, 881-904, 2003.

[8] Chan T. F and Tai X. C., Level set and total variation regularization for elliptic inverse problems with discontinuous coefficients, J. Comput. Phys. 193, 40-66, 2004.

[9] Chavent G., Nonlinear Least Squares for Inverse Problems: Theoretical Foundations and Step-by-Step Guide for Applications, New York: Springer, 2009. 
[10] Chavent G. and Kunisch K., The output least squares identifiability of the diffusion coefficient from an $H^{1}$-observation in a 2-D elliptic equation, ESAIM Control Optim. Calc. Var. 8, 423-440, 2002.

[11] Chicone C. and Gerlach J., A note on the identifiability of distributed parameters in elliptic equations, SIAM J. Math. Anal. 18, 1378-1384, 1987.

[12] Ciarlet P. G., Basis Error Estimates for Elliptic Problems, Handbook of Numerical Analisis, Vol. II, Ciarlet P. G. and Lions J.-L, eds., Amsterdam: Elsevier, 1991.

[13] Clément P., Approximation by finite element functions using local regularization, RAIRO Anal. Numér. 9, 77-84, 1975.

[14] Deckelnick K. and Hinze M., Convergence and error analysis of a numerical method for the identification of matrix parameters in elliptic PDEs, Inverse Problems 28, 15pp, 2012.

[15] Engl H. W., Hanke M. and Neubauer A., Regularization of Inverse Problems: Mathematics and its Applications, Dordrecht: Kluwer, 1996.

[16] Engl H. W., Kunisch K. and Neubauer A., Convergence rates for Tikhonov regularization of nonlinear ill-posed problems, Inverse Problems 5, 523-540, 1989.

[17] Falk R., Error estimates for the numerical identification of a variable coefficient, Math. Comput. 40, $537-546,1983$.

[18] Hanke M., A regularizing Levenberg-Marquardt scheme, with applications to inverse groundwater filtration problems, Inverse Problems 13, 79-95, 1997.

[19] Hào D. N. and Quyen T. N. T., Convergence rates for Tikhonov regularization of coefficient identification problems in Laplace-type equations, Inverse Problems 26, 23pp, 2010.

[20] Hào D. N. and Quyen T. N. T., Convergence rates for Tikhonov regularization of a two-coefficient identification problem in an elliptic boundary value problem, Numer. Math. 120, 45-77, 2012.

[21] Hein T. and Meyer M., Simultaneous identification of independent parameters in elliptic equationsnumerical studies, J. Inv. Ill Posed Probl. 16, 417-433, 2008.

[22] Hinze M., A variational discretization concept in control constrained optimization: the linear- quadratic case, Comput. Optim. Appl. 30, 45-61, 2005.

[23] Hinze M., Kaltenbacher B. and Quyen T. N. T., Identifying conductivity in electrical impedance tomography with total variation regularization, Numerische Mathematik, 43pp, 2017 (available https://doi.org/10.1007/s00211-017-0920-8).

[24] Hinze M. and Quyen T. N. T., Matrix coefficient identification in an elliptic equation with the convex energy functional method. Inverse problems 32, 29pp, 2016.

[25] Hoffmann K. H. and Sprekels J., On the identification of coefficients of elliptic problems by asymptotic regularization, Numer. Funct. Anal. Optim. 7, 157-177, 1985.

[26] Hsiao G. C. and Sprekels J., A stability result for distributed parameter identification in bilinear systems, Math. Methods Appl. Sci. 10, 447-456, 1988.

[27] Ito K. and Kunisch K., Lagrange Multiplier Approach to Variational Problems and Applications, Philadelphia: SIAM, 2008.

[28] Jin B., Khan T., Maass P. and Pidcock M., Function spaces and optimal currents in impedance tomography, J. Inv. Ill-Posed Problems. 19, 25-48, 2011.

[29] Isakov V., Inverse Source Problems, Rhode-Island: American Mathematical Society, 1989.

[30] Kaltenbacher B. and Schöberl J., A saddle point variational formulation for projection-regularized parameter identification, Numer. Math. 91, 675-697, 2002.

[31] Kelley C. T., Iterative Methods for Optimization, Philadelphia. SIAM, 1999. 
[32] Keung Y. L. and Zou J., An efficient linear solver for nonlinear parameter identification problems, SIAM J. Sci. Comput 22, 1511-1526, 2000.

[33] Knowles I., Uniqueness for an elliptic inverse problem, SIAM J. Appl. Math. 59, 1356-1370, 1999.

[34] Knowles I. and LaRussa M. A., Conditional well-posedness for an elliptic inverse problem, SIAM J. Appl. Math. 71, 952-971, 2011.

[35] Knowles I. and Wallace R., A variational method for numerical differentiation, Numer. Math. 70, 91$110,1995$.

[36] Kohn R. V. and Lowe B. D., A variational method for parameter identification, RAIRO Modél. Math. Anal. Numér. 22, 119-158, 1988.

[37] Kohn R. V. and Vogelius M., Determining conductivity by boundary measurements, Comm. Pure Appl. Math. 37, 289-298, 1984.

[38] Pechstein C., Finite and Boundary Element Tearing and Interconnecting Solvers for Multiscale Problems, Heidelberg New York Dordrecht London: Springer, 2010.

[39] Rannacher R. and Vexler B., A priori error estimates for the finite element discretization of elliptic parameter identification problems with pointwise measurements, SIAM J. Control Optim. 44, 1844-1863, 2005.

[40] Richter G. R., An inverse problem for the steady state diffusion equation, SIAM J. Appl. Math. 41, 210-221, 1981.

[41] Rudin L. I., Osher S. J. and Fatemi E., Nonlinear total variation based noise removal algorithms, Physica D 60, 259-268, 1992.

[42] Ruszczyński A., Nonlinear Optimization, Princeton: Princeton University Press, 2006.

[43] Schuster T., Kaltenbacher B., Hofmann B. and Kazimierski K. S., Regularization Methods in Banach Spaces, Berlin: Walter de Gruyter, 2012.

[44] Scott R. and Zhang S. Y., Finite element interpolation of nonsmooth function satisfying boundary conditions, Math. Comp. 54, 483-493, 1990.

[45] Sun N.-Z., Inverse Problems in Groundwater Modeling, Dordrecht: Kluwer, 1994.

[46] Tarantola A., Inverse Problem Theory and Methods for Model Parameter Estimation, Philadelphia: SIAM, 2005.

[47] Tartar L., The General Theory of Homogenization, Berlin: Springer, 2009.

[48] Wang L. and Zou J., Error estimates of finite element methods for parameter identification problems in elliptic and parabolic systems, Discrete Contin. Dyn. Syst. Ser. B. 14, 1641-1670, 2010. 